\title{
$\mathrm{N}-\mathrm{F}$ 键调控的高能量密度化合物合成与性能研究进展
}

\author{
翟连杰 ${ }^{a, b}$ 张俊林 ${ }^{a, b}$ 张家荣 ${ }^{a, b}$ 吴敏杰 ${ }^{a, b}$ \\ 毕福强*, $a, b$ 王伯周*, $a, b$ \\ ( ${ }^{a}$ 西安近代化学研究所 西安 710065) \\ ( ${ }^{b}$ 氟氮化工资源高效开发与利用国家重点实验室 西安 710065)
}

\begin{abstract}
摘要 与传统 $\mathrm{N}-\mathrm{O}$ 含能材料相比, $\mathrm{N}-\mathrm{F}$ 键调控的含能化合物因具有密度大、比冲高、与高热值金属燃烧放热量大等 独特优势而受到各国研究人员的广泛关注. 依据结构特点, $\mathrm{N}-\mathrm{F}$ 键含能化合物涵盖氟氮唑类含能化合物、二氟氨基二 硝甲基化合物、偕二氟氨基化合物以及二氟氨基聚合物等 4 类含能化合物. 综述了 $\mathrm{N}-\mathrm{F}$ 键调控的含能化合物研究进 展, 重点探讨了 $\mathrm{N}-\mathrm{F}$ 单键和二氟氨基引入策略以及其相关含能衍生物的合成技术途径, 评述了典型氟氮含能化合物 物化与爆轰性能. 发展高效、安全、便捷的 $\mathrm{N}-\mathrm{F}$ 键和二氟氨基合成方法, 开发结构稳定、感度适中的新型 $N$-氟唑类 含能化合物、偕二氟氨基硝胺环状等高能量密度化合物将是含能材料领域重要的发展方向之一.
\end{abstract}

关键词＼cjkstart含能材料; $\mathrm{N}-\mathrm{F}$ 键化合物; 二氟氨基; 合成; 爆轰性能

\section{Progress in Synthesis and Properties of High Energy Density Compounds Regulated by N-F Bond}

\author{
Zhai, Lianjie ${ }^{a, b}$ Zhang, Junlin ${ }^{a, b}$ \\ Zhang, Jiarong ${ }^{a, b}$ \\ Wu, Minjie $\mathrm{e}^{a, b}$ \\ Bi, Fuqiang*,a,b \\ Wang, Bozhou*,a,b \\ ( ${ }^{a}$ Xi'an Modern Chemistry Research Instritute, Xi'an 710065) \\ ( ${ }^{b}$ State Key Laboratory of Fluorine \& Nitrogen Chemical, Xi'an 710065)
}

\begin{abstract}
Compared with the traditional $\mathrm{N}-\mathrm{O}$ bond-based energetic materials, $\mathrm{N}-\mathrm{F}$ bond-regulated energetic materials have received worldwide research interest due to their special features of high density, high specific impulse, and high heat release upon combustion with metals. The $\mathrm{N}-\mathrm{F}$ bond-regulated energetic materials are classified as $\mathrm{N}-\mathrm{F}$ azoles, (difluoroamino)dinitromethyl-substituted derivatives, gem-bis(difluoramino)-substituted derivatives, and difluoroamino polymers according to their structural characteristics. The recent developments of $\mathrm{N}-\mathrm{F}$ bond and difluoramino $\left(\mathrm{NF}_{2}\right)$ energetic derivatives are reviewed. The construction methodologies of $\mathrm{N}-\mathrm{F}$ bond and difluoroamino groups as well as the synthetic routes to their energetic derivatives are emphatically reviewed. Moreover, the physicochemical and energetic properties of some typical compounds are briefly introduced. It will be the inevitable trend to develop effective, safe, and simple $\mathrm{N}-\mathrm{F}$ bond and difluoraminiation preparation method, and novel high-performing $\mathrm{N}-\mathrm{F}$ bond-based azoles and cyclic difluoramino-nitramines with moderate sensitivities and stable structures.
\end{abstract}

Keywords energetic materials; $\mathrm{N}-\mathrm{F}$ bond compounds; difluoramino group; synthesis; detonation performances

追求密度更大、能量更高的含能化合物始终是含能 材料领域的永恒主题. 自 1771 年英国人沃尔夫发明了 世界首个合成炸药一一三硝基苯酚(苦味酸)以来, 各国 合成化学家在含能材料前进的道路上从未此步 ${ }^{[1 \sim 4]}$. 从 第一代含能材料的三硝基甲苯(TNT), 到以六硝基六氮 杂异伍兹烷(CL-20)为代表的第三代含能材料, 其核心
思想与策略都是在 $\mathrm{C} 、 \mathrm{H} 、 \mathrm{~N}$ 等元素构成的链状、环状 及笼型骨架中引入硝基基团，包括硝胺基 $\left(\mathrm{NNO}_{2}\right)$ 、硝酸 酯基 $\left(\mathrm{ONO}_{2}\right)$ 和多硝甲基(二硝甲基、三硝甲基和氟二硝 甲基)等, 进而设计、合成新型高能量密度材料 ${ }^{[5 \sim 15]}$. 在 某种程度上，含能材料的发展史可以认为是硝基化合物 的开发史. 硝基不仅能大幅提高 $\mathrm{CHON}$ 化合物密度，更

* Corresponding authors. E-mail: bifuqiang@gmail.com; wbz600@163.com

Received January 12, 2020; revised February 27, 2020; published online March 6, 2020.

Project supported by the National Natural Science Foundation of China (No. 21805224).

国家自然科学基金资助项目(No. 21805224). 
重要的是硝基是分子内 “氧” 的来源, 在爆轰反应中, 硝 基中的氧将 $\mathrm{C} 、 \mathrm{H}$ 等氧化成稳定氧化态(水、一氧化碳、 二氧化碳等), 进而释放能量, 这也是含能材料与能源 材料如煤、石油和天然气的本质区别. 然而, 最近十几 年, 各国在开发能量密度达到或超过 CL-20 的新型高能 量密度化合物道路上却踌躇不前. 究其原因, 一方面, 向单个分子中引入更多数量的硝基等含能基团时, 尽管 密度和爆轰性能在一定程度上得到提升, 但同时也会导 致目标化合物合成步骤长、合成难度大、制造成本高、 感度高、分解温度低等问题，严重限制其应用前景 ${ }^{[16,17]}$; 另一方面, 以硝基为代表的 $\mathrm{CHON}$ 类含能化合物能量 密度存在理论上限, 高度对称的致密笼型高能化合物八 硝基立方烷(ONC) $)^{[18,19]}$ 和 CL-20 ${ }^{[20,21]}$ 的密度和爆轰性能 已达到很高的水平, 想要在此基础上进一步提高, 变得 愈发困难. 因此, 打破现有以硝基为基本能量单元的发 展策略, 突破 $\mathrm{CHON}$ 类含能材料能量瓶颈, 具有重要的 理论和实践意义.

氟是目前发现电负性最强的元素, 也是氧化能力最 强的非金属单质, 其原子半径也显著小于氧原子. 将 $\mathrm{N}-\mathrm{F}$ 键替代硝基中 $\mathrm{N}-\mathrm{O}$ 键形成的氟氮含能化合物具 有以下独特优势: (1)氟原子较小的原子半径赋予含氟含 能化合物更高的堆积密度, 二氟氨基化合物密度 $(\rho)$ 理 论上限可以达到 $2.303 \mathrm{~g} / \mathrm{cm}^{3}$, 显著高于对应硝基化合物 密度的理论上限 $2.166 \mathrm{~g} / \mathrm{cm}^{3}{ }^{[22]}$. (2) $\mathrm{N}-\mathrm{F}$ 键具有较低的 键能 $(280 \mathrm{~kJ} / \mathrm{mol})$, 氟氮含能物质在燃烧分解过程中形 成的气体产物 $\mathrm{H}-\mathrm{F}$ 键能高 $(565 \mathrm{~kJ} / \mathrm{mol})$, 相对分子质量 低, 生成热高, 有利于提高推进剂或炸药的燃气比容, 可大幅提高发射药、推进剂和混合炸药的能量水平 ${ }^{[23,24]}$. (3)高热值金属燃烧剂(铝粉、嗍粉等)氟化反应比相应氧 化反应放出更多的热量, 每摩尔铝氟化放热量是氧化放 热量的 1.80 倍, 每摩尔硼氟化放热量是其氧化放热量的

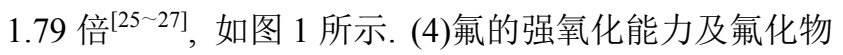
独特的低熔点特性能够显著改善高热值可燃金属元素 的燃烧或爆炸反应效率, 氧化铝的熔点高达 $2977{ }^{\circ} \mathrm{C}$, 燃烧过程中铝颗粒表面形成的难挥发氧化铝层严重制 约着铝粉的充分燃烧, 而氟化铝沸点仅为 $1275{ }^{\circ} \mathrm{C}$, 燃 烧高温能够轻易实现 “剥壳效应” ，大大提高金属粉的 燃烧效率. 研究表明, 基于 3,3,7,7-四(二氟氨基)八氢化1,5-二硝基-1,5-二氮杂辛烷(HNFX)、可燃剂 $\mathrm{BeH}_{2}$ 配伍 组成的推进剂配方, 理论比冲 $\left(I_{\mathrm{sp}}\right)$ 高达 $3038 \mathrm{~N} \cdot \mathrm{s} / \mathrm{kg}$, 显 示出诱人的应用前景 ${ }^{[28]}$.

将 $\mathrm{N}-\mathrm{F}$ 键引入含能材料的研究非常早, 并且多集 中在二氟氨基含能化合物的合成与反应特性研究 ${ }^{[29 ~ 32]}$. 早在 1936 年, Juff 等 ${ }^{[33]}$ 报道了首个有机二氟氨基化合物 二氟氨基三氟甲烷，引起了美国高级工程局和国防部

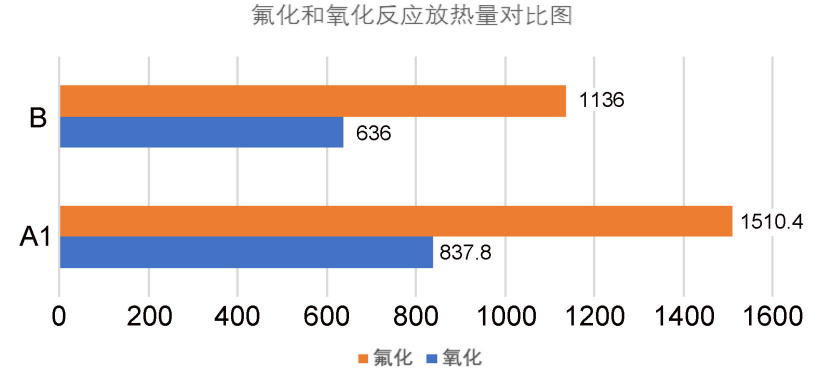

图 1 每摩尔嗍和铝氟化和氧化反应放热量

Figure 1 Fluorination and oxidation heat release of per mole of $\mathrm{B}$ and $\mathrm{Al}$

的关注. 鉴于 $\mathrm{N}-\mathrm{F}$ 含能化合物在开发推进剂用高性能 氧化剂上显示出的巨大优势，旨在探究其应用可行性的 “Principia”项目于 1958 1965 间实施 ${ }^{[34]}$. 在这期间，利 用 $\mathrm{F}_{2} 、 \mathrm{~N}_{2} \mathrm{~F}_{4}$ 和 $\mathrm{HNF}_{2}$ 等合成手段, 大量二氟氨基和偕二 氟氨基化合物被合成出来, 如 1,2-二(二氟氨基)乙烷 ${ }^{[35]}$ 、 $2,2-$ 二(二氟氨基) 乙醇 ${ }^{[36]} 、 2,3-$ 二(二氟氨基)硝酸丙酯 ${ }^{[37]}$ 、

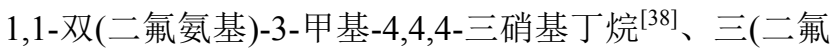
氨基)甲氧基-5,5,5-三硝基-3-丁硝胺 ${ }^{[39]}$ 等. 尽管如此，由 于当时合成的多数二氟氨基化合物结构中的 $\alpha-\mathrm{H}$ 与二 氟氨基极易脱去 HF 形成腈，导致该类材料普遍存在结 构稳定性差、感度高等问题, 致使相关的研究几乎停滞. 进入 20 世纪 90 年代以来，随着认识的深入和新型氟化 试剂的开发, 以 HNFX 为代表的新型氟氮含能化合物重 新引起各国学者的广泛关注, 美国海军空战武器中心 Chapman 教授和俄罗斯科学院相关人员在这方面做了 大量工作，陆续设计并合成出分子结构稳定的新型二氟 氨基含能化合物. 我国在氟氮含能化合物研究方面也有 诸多亮点, 西安近代化学研究所郑远洋等 ${ }^{[40]} 1988$ 年 在国内报道了首个氟氮高能化合物四(二氟氨基亚硝胺 基)乙烷，并完成了密度、感度、爆发点和爆速等性能的 研究. 近期, 南京理工大学、黎明化工研究院等单位在 氟氮含能粘合剂、增塑剂方面取得可喜成果 ${ }^{[41 ~ 43]}$. 但与 国外研究工作相比, 在研究的深度和广度方面, 还存在 一定差距

鉴于氟氮化合物作为含能材料的巨大潜力，本文全 面概述了近期氟氮含能化合物的合成研究进展，以氟氮 化合物结构特点进行分类，着重介绍了氮氟唑类、二氟 氨基二硝甲基化合物、偕二氟氨基化合物、二氟氨基聚 合物等的合成途径，并对典型氟氮含能化合物结构特点 和爆轰性能进行了梳理, 以期为进一步开展相关研究工 作提供参考.

\section{$1 \mathrm{~N}$ - F 键合成方法与试剂}

含有 $\mathrm{C}-\mathrm{F}$ 键的有机氟化物在许多尖端技术和重大 工业项目及医药、农药和催化工业中都有着广泛而深入 
的研究和应用, $\mathrm{C}-\mathrm{F}$ 键合成方法和氟化试剂发展较为 成熟. 与之相比, $\mathrm{N}-\mathrm{F}$ 键合成途径和策略相对较少, 主 要集中在氟气氟化和二氟氨基化两个方面. $\mathrm{N}-\mathrm{F}$ 键试 剂是氟氮含能化合物合成以及氟氮化合物性能研究的 基础, 故有必要对常见的 $\mathrm{N}-\mathrm{F}$ 键合成方法和试剂进行 简单介绍.

\section{$1.1 \mathrm{~F}_{2}$}

利用 $F_{2}$ 对相关底物进行氟化是合成氟氮化合物最 直接、最简便的方法, 也是氟氮化合物中氟的终极来源. $F_{2}$ 氟化主要通过以下 2 条途径生成相应的氟氮化合物: (1)通过对氮杂芳环氮负离子氧化, 生成相应 $\mathrm{N}-\mathrm{F}$ 键氮 杂芳环化合物 ${ }^{[44,45]}$; (2)对仲胺(尤其酰胺结构)、伯胺化合 物氟化生成二氟氨基化合物 ${ }^{[46 ~ 50]}$, 如 Scheme 1 所示. 其中, 对氨气、尿素等化合物氟化制备的小分子氟氮化 合物 $\mathrm{HNF}_{2} 、 \mathrm{~N}_{2} \mathrm{~F}_{4} 、 \mathrm{NF}_{3}$ 更是高效的二氟氨基化试剂, 利 用这些二氟氨基试剂能够衍生出数量众多、性能各异的 二氟氨基化合物.

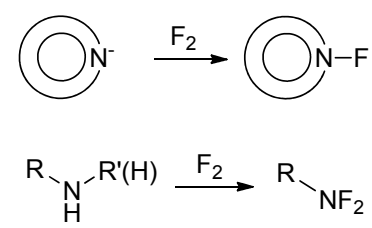

图式 $1 \mathrm{~F}_{2}$ 对含氮化合物的氟化反应

Scheme 1 Fluorination of nitrogen-containing compounds with $\mathrm{F}_{2}$

\section{$1.2 \mathrm{HNF}_{2}$}

$\mathrm{HNF}_{2}$ 被广泛应用于合成二氟氨基化合物、偕二氟 氨基化合物, 是一种重要的二氟氨基试剂和中间体. $\mathrm{HNF}_{2}$ 不稳定, 对撞击、摩擦均很敏感, 在结晶和融化过 程中易发生爆炸 ${ }^{[51,52]} . \mathrm{HNF}_{2}$ 合成方法有多种, 包括 $\mathrm{N}_{2} \mathrm{~F}_{4}$ 法 $^{[53]}$ 、三苯基二氟氨基甲烷法 $\left.\left[(\mathrm{Ph})_{3} \mathrm{CNF}_{2}\right)\right]^{[54,55]}$ 和尿素 法 ${ }^{[56,57]}$, 其中通过尿素氟化、水解合成的方法最为常见, 路线如 Scheme 2.

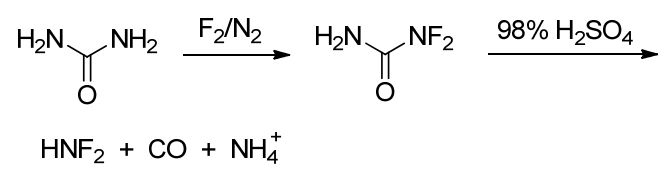

图式 $2 \mathrm{HNF}_{2}$ 的合成

Scheme 2 Synthesis of $\mathrm{HNF}_{2}$

$\mathrm{HNF}_{2}$ 不仅能与具有羰基结构的醛、酮发生亲核加 成反应 ${ }^{[58,59]}$, 生成相应的偕二氟氨基化合物, 还能与不 饱和烯、炔反应 ${ }^{[60]}$, 生成相应二氟氨基化合物, 如 Scheme 3 所示. $\mathrm{HNF}_{2}$ 与羰基反应如 Scheme 4 所示, 在 强酸条件下, 羰基上氧质子化, 二氟化氨与羰基发生加
成反应得到醇中间体，随后经羟基质子化脱水、二氟化 氨再次亲核进攻，生成偕二氟氨基化合物.

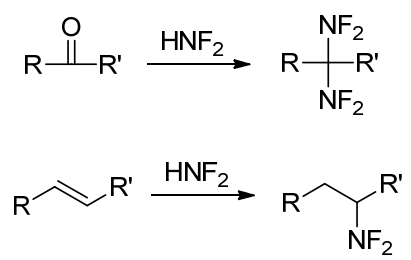

图式 $3 \mathrm{HNF}_{2}$ 与羰基和烯烃化合物的反应

Scheme 3 Reactions of $\mathrm{HNF}_{2}$ with carbonyl compounds and alkenes

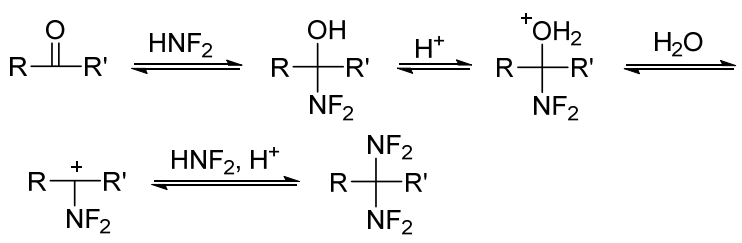

图式 $4 \mathrm{HNF}_{2}$ 与羰基化合物反应机理

Scheme 4 Mechanism of difluoramination of carbonyl compounds

\section{$1.3 \quad \mathrm{~F}_{2} \mathrm{NSO}_{3} \mathrm{H}$ 和 $\mathrm{F}_{2} \mathrm{NSO}_{3} \mathrm{Na}$}

$\mathrm{HNF}_{2}$ 常温下为气体且危险性较高, 故开发安全性 能好、常温下为液体或固体的二氟氨基化试剂显得非常 必要. 1966 年, Keith 等 ${ }^{[61]}$ 利用 $\mathrm{HNF}_{2}$ 与 $\mathrm{SO}_{3}$ 反应，合成了 室温下为液体的二氟氨基试剂 $\mathrm{F}_{2} \mathrm{NSO}_{3} \mathrm{H}$. 尽管热稳定性 和安全性相对 $\mathrm{HNF}_{2}$ 有较大的提升, 但 $\mathrm{F}_{2} \mathrm{NSO}_{3} \mathrm{H}$ 在室温 下仍会缓慢分解，生成 $\mathrm{N}_{2} \mathrm{~F}_{4} 、 \mathrm{SO}_{2}$ 和 $\mathrm{H}_{2} \mathrm{SO}_{4} .2006$ 年, Haiges 等 ${ }^{[62]}$ 以 $\mathrm{NaSO}_{3} \mathrm{NH}_{2}$ 为原料, 通过 $\mathrm{F}_{2}$ 直接氟化, 成 功获得了 $\mathrm{F}_{2} \mathrm{NSO}_{3} \mathrm{Na}$, 收率达到 $94 \%$ (Scheme 5). $\mathrm{F}_{2} \mathrm{NSO}_{3} \mathrm{Na}$ 室温下为固体, 在干燥的环境中能够稳定存 在, 其在合成方法简易程度、安全性等方面, 较 $\mathrm{HNF}_{2}$ 、 $\mathrm{F}_{2} \mathrm{NSO}_{3} \mathrm{H}$ 均有较大提高. 与 $\mathrm{HNF}_{2}$ 相似, $\mathrm{F}_{2} \mathrm{NSO}_{3} \mathrm{H}$ 和 $\mathrm{F}_{2} \mathrm{NSO}_{3} \mathrm{Na}$ 均能与酮、醛化合物生成相应的偕二氟氨基 化合物 ${ }^{[63 \sim 65]}$

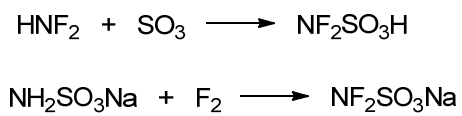

图式 $5 \mathrm{~F}_{2} \mathrm{NSO}_{3} \mathrm{H}$ 和 $\mathrm{F}_{2} \mathrm{NSO}_{3} \mathrm{Na}$ 的合成 Scheme 5 Synthesis of $\mathrm{F}_{2} \mathrm{NSO}_{3} \mathrm{H}$ and $\mathrm{F}_{2} \mathrm{NSO}_{3} \mathrm{Na}$

\section{$1.4 \mathrm{Ph}_{3} \mathrm{CNF}_{2}$}

$\mathrm{Ph}_{3} \mathrm{CNF}_{2}{ }^{[54,55,66]}$ 是一种室温下稳定的固态二氟氨基 化试剂, 其熔点为 $82{ }^{\circ} \mathrm{C} \sim 83{ }^{\circ} \mathrm{C}$, 且在空气中不潮解. $\mathrm{Ph}_{3} \mathrm{CNF}_{2}$ 合成路线如 Scheme 6 所示，以三苯基氯甲烷为 原料, 与 $\mathrm{N}_{2} \mathrm{~F}_{4}$ 反应生成 $\mathrm{Ph}_{3} \mathrm{CNF}_{2}$, 收率可达 $88 \%$. $\mathrm{Ph}_{3} \mathrm{CNF}_{2}$ 在浓硫酸中可分解成三苯甲基正离子和 $\mathrm{HNF}_{2}$, 故 $\mathrm{Ph}_{3} \mathrm{CNF}_{2}$ 在强酸下也能与酮反应, 生成相应的偕二氟 氨基化合物. 因此, $(\mathrm{Ph})_{3} \mathrm{CNF}_{2}$ 可作为室温稳定、安全、 
可操作性强的二氟氨基化试剂, 具有广阔应用空间. 唯 一不足的是, 合成 $\mathrm{Ph}_{3} \mathrm{CNF}_{2}$ 过程中仍需要使用危险性高 的 $\mathrm{N}_{2} \mathrm{~F}_{4}$ 以及有毒金属录.

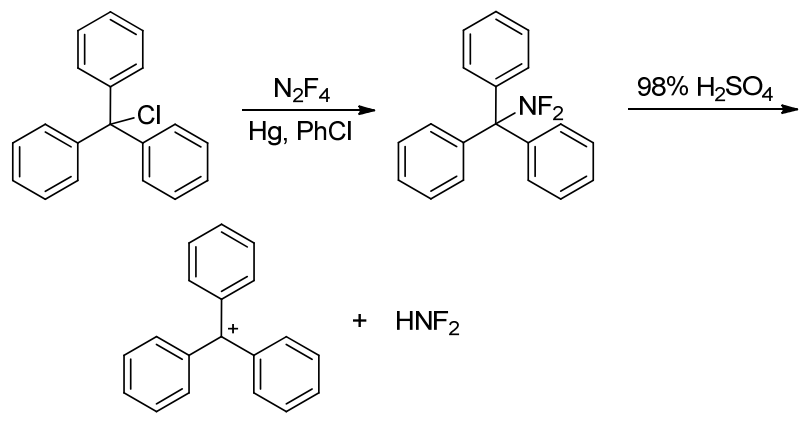

图式 $6 \mathrm{Ph}_{3} \mathrm{CNF}_{2}$ 的合成及其与浓硫酸反应

Scheme 6 Synthesis of $\mathrm{Ph}_{3} \mathrm{CNF}_{2}$ and its reaction with conc. $\mathrm{H}_{2} \mathrm{SO}_{4}$

\section{$1.5 \mathrm{~F}_{2} \mathrm{NOSO}_{2} \mathrm{~F}$}

$\mathrm{F}_{2} \mathrm{NOSO}_{2} \mathrm{~F}$ 是一种具有强氧化性的氟化试剂, 通过 与碳、氮阴离子化合物发生氧化反应, 生成相应的二氟 氨基化产物. 1963 年, Lustig 等 ${ }^{[67]}$ 通过 $\mathrm{S}_{2} \mathrm{O}_{6} \mathrm{~F}_{2}$ 与 $\mathrm{N}_{2} \mathrm{~F}_{4}$ 之 间自由基反应, 首次合成了 $\mathrm{F}_{2} \mathrm{NOSO}_{2} \mathrm{~F}$, 并完成了结构 和部分物化性能测试. $\mathrm{F}_{2} \mathrm{NOSO}_{2} \mathrm{~F}$ 熔点为 $-128.8{ }^{\circ} \mathrm{C}$, 沸 点为 $-2.5{ }^{\circ} \mathrm{C}$, 液体密度为 $1.5 \mathrm{~g} / \mathrm{cm}^{3}$. 目前, 文献已利 用 $\mathrm{F}_{2} \mathrm{NOSO}_{2} \mathrm{~F}$ 与二硝甲基阴离子化合物、唑阴离子化合 物反应, 成功合成了二氟氨基二硝甲基化合物和 $N-$ $\mathrm{NF}_{2}$ 唑类化合物 ${ }^{[68 \sim 71]}$, 该试剂已成为构建二氟氨基高能 化合物的重要手段, 如 Scheme 7 所示.

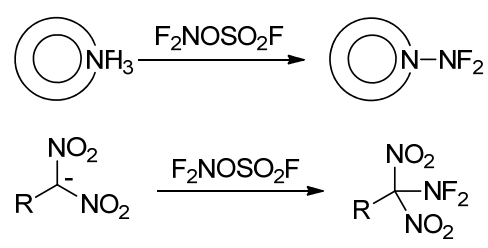

图式 $7 \mathrm{~F}_{2} \mathrm{NSO}_{3} \mathrm{~F}$ 与负离子化合物二氟氨基化反应 Scheme 7 Difluoramination of anion compounds with $\mathrm{F}_{2} \mathrm{NSO}_{3} \mathrm{~F}$

\section{$1.6 \mathrm{~N}_{2} \mathrm{~F}_{4}$}

$\mathrm{N}_{2} \mathrm{~F}_{4}$ 是一种无色气体状态的全氟氮化合物 ${ }^{[72,73]}$, 其 制备方法有多种, 但主要以 $\mathrm{HNF}_{2}$ 氧化和 $\mathrm{NF}_{3}$ 与金属氟 化反应两种方法制备 ${ }^{[51]}$, 如 Scheme 8 所示. $\mathrm{N}_{2} \mathrm{~F}_{4}$ 分子中 $\mathrm{N}-\mathrm{N}$ 单键较长 $(0.14 \mathrm{~nm})$, 键能较低, $\mathrm{N}-\mathrm{N}$ 单键解离能 介于 $80 \sim 91 \mathrm{~kJ} / \mathrm{mol}$ 之间, 很容易生成二氟氨自由基 ${ }^{[31]}$. 二氟氨自由基反应活性高, 能与烯烃、炔烃等不同类型 结构的底物反应, 且产物复杂, 也是制备二氟氨基化合 物的重要途径 ${ }^{[35,74 ~ 76]}$, Scheme 9 列举了部分示例.

除了上述介绍试剂外, $\mathrm{CsOSO}_{2} \mathrm{OF}^{[77]}$ 、 Select-

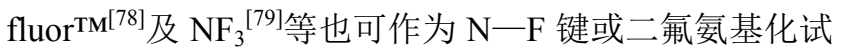
剂, 但限于篇幅, 不在赘述.

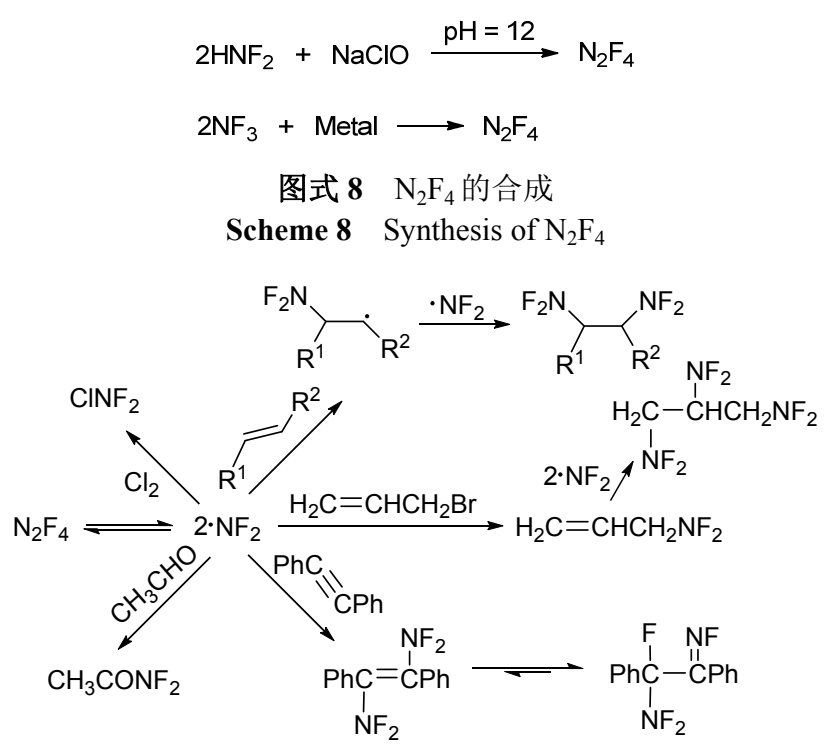

图式 $9 \mathrm{~N}_{2} \mathrm{~F}_{4}$ 发生的典型反应

Scheme 9 Typical reaction of $\mathrm{N}_{2} \mathrm{~F}_{4}$

\section{2 氟氮唑类含能化合物}

\section{$2.1 \mathrm{~N}-\mathrm{F}$ 唑类含能化合物}

唑环(包括咪唑、吡唑、三唑、四唑等)化合物具有 氮含量高、生成焓大、爆轰性能优异和相对钝感等特性, 在新型炸药、低特征信号推进剂、气体发生剂等领域具 有广泛的应用前景. 将唑环上氢用氟取代后会有以下好 处: (1)消除唑类化合物酸性, 改善其相容性; (2)化合物 的密度、氧平衡和生成焓进一步提高, 进而提升化合物 爆速和爆压; (3)唑环芳香性能够与 $\mathrm{N}-\mathrm{F}$ 键形成共轭体 系, 进而增加结构稳定性.

Forohar 等 ${ }^{[44]}$ 报道了硝基咪唑化合物氮氟化反应, 以 4-硝基咪唑为原料, 在浓硝酸和乙酸䣶体系下对其硝 化合成 2,4-二硝基咪唑中间体，再使用 5\% $\mathrm{F}_{2}$ 直接对其 氟化，获得了室温稳定的氟化产物 2,4-二硝基氟化咪唑 (1), 合成路线如 Scheme 10 所示. 但后续关于该化合物 的密度、热稳定性、感度及爆轰等进一步性能数据并未 见报道.

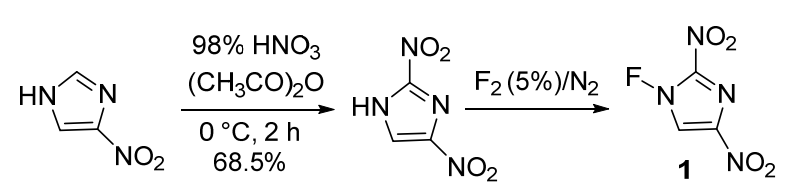

图式 10 化合物 1 的合成

Scheme 10 Synthesis of compound 1

Dalinger 等 ${ }^{[45]}$ 尝试在甲醇或乙腈溶液中使用过量的 $\mathrm{CsOSO}_{2} \mathrm{~F}$ 对多硝基联吡唑、多硝基并吡唑等的钠盐进行 氟化, 期望得到吡唑氮氟化物, 但反应过程中并未检测 到相关产物. 实验发现, 反应结束后 $\mathrm{CsOSO}_{2} \mathrm{~F}$ 已全部消 
耗, 且溶液呈酸性, 作者认为 $\mathrm{CsOSO}_{2} \mathrm{~F}$ 首先与溶剂发生 氟化反应, 生成 HF 并将吡唑钠酸化成很难被氟化的 $\mathrm{N}-\mathrm{H}$ 结构, 导致氟化失败. 随后该课题组使用 $10 \% \mathrm{~F}_{2}$ 对相应吡唑钠盐进行氟化, 为了减少副反应及 $\mathrm{HF}$ 带来 不利因素, 反应加入 $\mathrm{NaF}$ 作为 $\mathrm{HF}$ 吸收剂, 在 $-60 \sim-$ $70{ }^{\circ} \mathrm{C}$ 低温条件下成功获得相应氟化物, 其中 3,3 ',5,5'四硝基-1H,1'H-4,4'-联吡唑(2)收率最高，达到 66\%，其 他介于 $18 \% \sim 50 \%$ 之间, 合成路线如 Scheme 11 所示. 热分析结果显示, 合成的吡唑氮氟化产物分解点普遍较 高, 其中 1, $1^{\prime}$-二氟-3,3',4,4'-四硝基- $1 H, 1^{\prime} H$-5, 5'-联吡唑 (3)和 1,4-二氟-3,6-二硝基吡唑[4,3-c]并吡唑(4)热分解温 度高于 $190{ }^{\circ} \mathrm{C}$, 表明 $\mathrm{N}-\mathrm{F}$ 键的引入并没有大幅降低, 分子结构稳定性. 目前, 多硝基唑类化合物氮氟化及氮 氟化产物性能的文献较少, 该研究为后续相关研究提供 了参考.
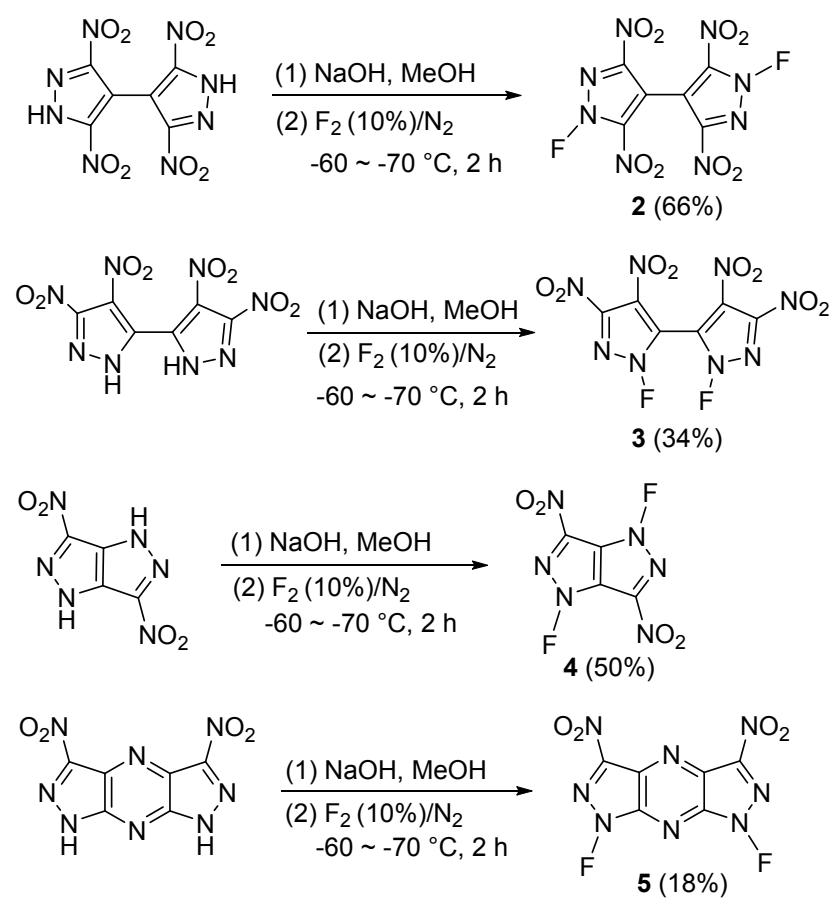

图式 11 硝基吡唑 $N-\mathrm{F}$ 衍生物的合成

Scheme 11 Synthesis of $N-\mathrm{F}$ derivatives of nitrated pyrazoles

\section{$2.2 \mathrm{~N}-\mathrm{NF}_{2}$ 唑类含能化合物}

为了进一步提升 $\mathrm{N}-\mathrm{F}$ 键在唑类含能化合物中的结 构占比, Dalinger 等 ${ }^{[80]}$ 以多硝基取代的咪唑、吡唑和三 唑盐为原料, 乙腈为溶剂, PEG-400 作为相转移助剂, 使用二氟氨基试剂 $\mathrm{F}_{2} \mathrm{NOSO}_{2} \mathrm{~F}$, 合成了咪唑、吡唑和三 唑的 $\mathrm{N}-\mathrm{NF}_{2}$ 化合物, 其中化合物 9, 10, 12, 17 极度不稳 定, 并未完成分离表征, 其收率根据 ${ }^{19} \mathrm{~F}$ NMR 推测得到, 合成路线如 Scheme 12 所示. 由于母体结构的不同, 这 些硝基唑的 $\mathrm{N}-\mathrm{NF}_{2}$ 化收率差异较大, 介于 $5 \% \sim 80 \%$ 之 间.
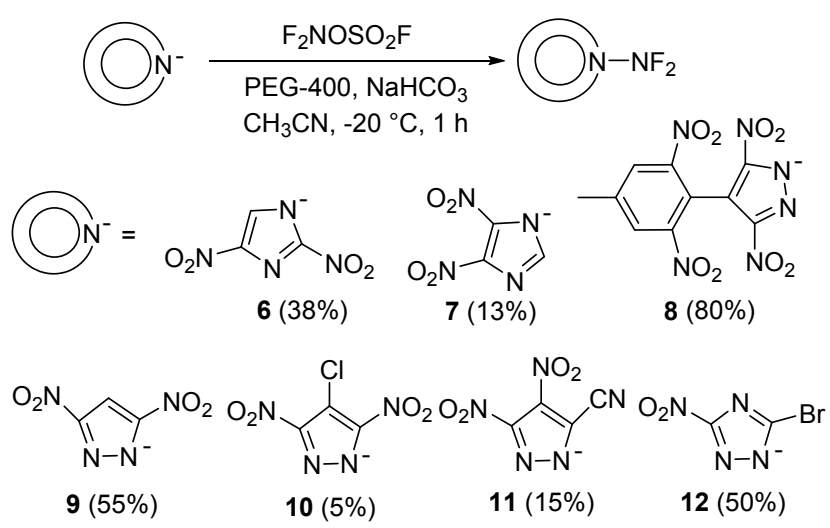

$\mathrm{O}_{2} \mathrm{~N}_{\mathrm{K}^{\mathrm{N}}-\mathrm{N}^{-}}^{\mathrm{N}} \mathrm{Y}^{\text {coome }}$

$13(22 \%)$<smiles>CC(=O)n1ncnc1-c1nc([N+](=O)[O-])n[nH]1</smiles>

$16(32 \%)$<smiles>O=[N+]([O-])c1n[nH]c(-c2[nH]nc([N+](=O)[O-])c2[N+](=O)[O-])c1[N+](=O)[O-]</smiles>

$14(22 \%)$<smiles>O=[N+]([O-])c1n[nH]c(-n2cncn2)n1</smiles>

$15(11 \%)$
图式 $12 N-\mathrm{NF}_{2}$ 唑类化合物的合成

Scheme 12 Synthesis of $N$-difuoroaminoazoles

表 1 列举了部分化合物的热分解温度 $\left(T_{\mathrm{dec}}\right)$ 、密度和 爆轰性能，并与黑索金(RDX)进行了对比. 由于二氟氨 基的引入，该类化合物密度普遍较高，化合物 6.7 和 14 的密度均超过 $1.90 \mathrm{~g} / \mathrm{cm}^{3}$. 但与 Scheme 11 中吡唑氮氟 化产物相比，该类化合物热稳定性普遍较差，化合物 11、14 和 15 的分解温度不超过 $100{ }^{\circ} \mathrm{C}$. 化合物 6 密度 较高、氧平衡较好, 故其爆速 $\left(V_{\mathrm{det}}\right)$ 达到 $8500 \mathrm{~m} / \mathrm{s}$, 爆压 $(P)$ 超过 $30 \mathrm{GPa}$. 另外，化合物 $\mathbf{1 4}$ 的单晶结构表明 $\mathrm{N}-\mathrm{F}$ 和 $\mathrm{N}_{\mathrm{ring}}-\mathrm{N}_{\text {difuoroamino }}$ 平均键长分别为 0.1367 和 0.1410 $\mathrm{nm}, \mathrm{F}-\mathrm{N}-\mathrm{F}$ 和 $\mathrm{N}-\mathrm{N}-\mathrm{F}$ 平均键角为 $101.5^{\circ}$ 和 $104.2^{\circ}$, $\mathrm{N}-\mathrm{NF}_{2}$ 键长较长, 解离能较低, 解释了该类化合物较 高的感度和低的热分解温度的原因。

表 $1 \mathrm{~N}-\mathrm{NF}_{2}$ 唑类化合物与 RDX、HMX 的性能对比 Table 1 Properties of $\mathrm{N}$-difluoroaminoazoles compared with RDX and HMX

\begin{tabular}{crcrrr}
\hline Substrate & $T_{\text {dec }}{ }^{\circ} \mathrm{C}$ & $\Delta H_{\mathrm{f}} /\left(\mathrm{kJ} \cdot \mathrm{mol}^{-1}\right)$ & $\rho /\left(\mathrm{g} \bullet \mathrm{cm}^{-3}\right)$ & $V_{\mathrm{det}} /\left(\mathrm{m} \bullet \mathrm{s}^{-1}\right)$ & $P / \mathrm{GPa}$ \\
\hline $\mathbf{6}$ & 70 & 117.2 & 1.92 & 8536 & 31.4 \\
$\mathbf{7}$ & 62 & 118.4 & 1.91 & 8494 & 31.0 \\
$\mathbf{1 0}$ & 127 & 228.0 & 1.80 & 7925 & 27.4 \\
$\mathbf{1 4}$ & 100 & 421.7 & 1.92 & 8257 & 30.9 \\
$\mathbf{1 5}$ & 84 & 458.8 & 1.88 & 8462 & 30.0 \\
$\mathrm{RDX}$ & 204 & 67 & 1.82 & 8850 & 34.9 \\
\hline
\end{tabular}

\section{$2.3 \mathrm{C}-\mathrm{NF}_{2}$ 唑类含能化合物}

Fokin 等 ${ }^{[81]}$ 以 1-甲基-5-氨基四唑、2-甲基-5-氨基四 唑为原料, 在 $\mathrm{NaF}$ 的乙腈溶液中, 利用 $\mathrm{F}_{2}$ 直接对其氟化, 
成功获得了 5-二氟氨基-1-甲基四唑(18)、5-二氟氨基-2甲基四唑(19), 收率分别为 37\% 和 23.7\%, 如 Scheme 13 所示. 这是首次将二氟氨基引入到四唑结构中, 且得到 的目标物能在常规条件下稳定存在. 随后, 为了将二氟 氨基引入到四唑烷烃基上，该课题组又使用二氟化氨 $\left(\mathrm{HNF}_{2}\right)$ 对 2-羟甲基-5-硝基四唑进行氟化, 得到了 2-二 氟氨甲基-5-硝基四唑(20), 收率为 $22 \%$. 上述研究成功 实现了向四唑环上碳以及四唑上氮甲基上引入二氟氨 基，丰富了氟氮唑含能化合物种类.

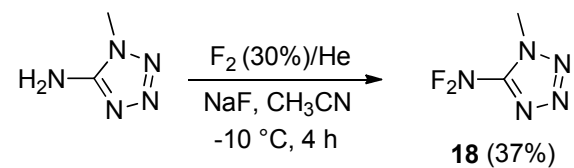

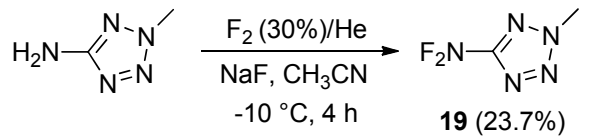<smiles>O=[N+]([O-])c1nnn(CO)n1</smiles>

图式 $13 \mathrm{C}-\mathrm{NF}_{2}$ 四唑化合物的合成

Scheme 13 Synthesis of $\mathrm{C}-\mathrm{NF}_{2}$-substituted tetrazoles

Shreeve 等 ${ }^{[82]}$ 以乙烯与 $\mathrm{N}_{2} \mathrm{~F}_{4}$ 为原料, 在高温和氟化 钾作用下合成了二氟氨基二氟乙腈 $(21)$, 由于氰基较好 的反应活性, 可以以此衍生出多种二氟氨甲基取代唑类 化合物, 如 Scheme 14 所示. 该课题组 ${ }^{[83,84]}$ 通过化合物
21 与氨发生加成反应，合成了氨化产物 23 , 该化合物高 温加热后聚合成二氟氨甲基取代的均三嗪化合物 24 . 同样, 中间体 21 与羟胺反应, 获得了中间体 $\mathbf{2 5}$, 将其与 酸酐或光气反应, 在高温下脱 $\mathrm{H}_{2} \mathrm{O}$ 或 $\mathrm{HCl}$ 后, 环化成二 氟氨甲基取代的异呋咱化合物 26、29 和 30. 随后，该课 题组 ${ }^{[85]}$ 通过中间体 21 与叠氮化钠发生环化反应, 高收 率得到二氟氨基二氟甲基四唑钠盐化合物 22. 然而, 相 关化合物的密度、热性能、感度及爆轰性能并未见报道.

鉴于四唑钠盐 22 具有较高生成焓及较好的能量水 平, 2007 年 Shreeve 课题组 ${ }^{[86]}$ 通过钠盐 22 酸化获得二氟 氨基二氟甲基四唑(31), 利用四唑酸性，与氨水成盐得 到其铵盐 32. 铵盐 32 与硝酸银作用生成银盐, 银盐与 有机富氮盐 1,5-二氨基-4-甲基四唑和 1-甲基-4-氨基1,2,4-三唑的碘化物发生复分解反应, 生成了含二氟氨 基的四唑富氮含能盐 33 34 (Scheme 15). 与化合物 33 和 34 相比, 铵盐 22 能量性能最好, 其实测密度达到 $1.88 \mathrm{~g} / \mathrm{cm}^{3}$, 热分解温度为 $158{ }^{\circ} \mathrm{C}$, 理论爆速和爆压分 别为 $8490 \mathrm{~g} / \mathrm{cm}^{3}$ 和 $28.8 \mathrm{GPa}$, 显示出优异的爆轰性能.

\section{3 二氟氨基二硝甲基含能化合物}

在现有母体中引入新型高能基团是提升含能材料能量 水平的有效途径. 二氟氨基二硝甲基是一类结构稳定、 能量较高的新型高能基团，相比二硝甲基，二氟氨基的 引入不仅能够显著提高能量水平，还能消除二硝甲基的 酸性; 与三硝甲基相比，二氟氨基更小的空间占位有望 实现更好的结构稳定性和更大的堆积密度.

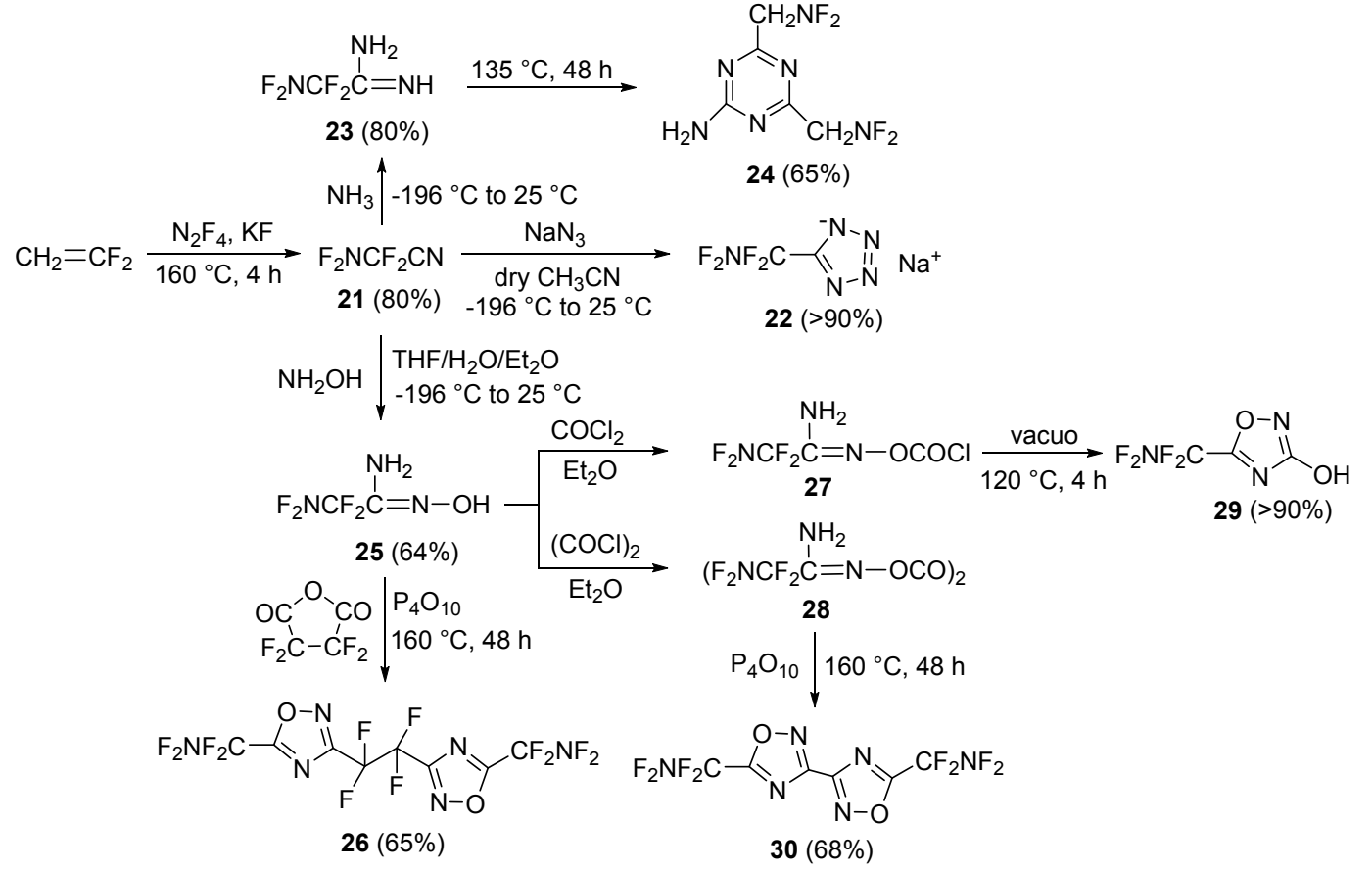

图式 14 二氟氨甲基化合物的合成

Scheme 14 Synthesis of difuoroaminomethyl-substituted compounds 


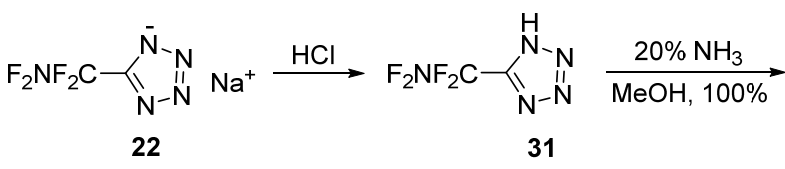<smiles></smiles>

图式 15 二氟氨甲基四唑及其含能盐的合成

Scheme 15 Synthesis of difuoroaminomethyl-substituted tetrazole and its energetic salts

\section{1 二氟氨基二硝甲基链状烷烃化合物}

多硝基烷烃含能化合物具有种类丰富、研究起始时 间早的特点, 将二氟氨基二硝甲基引入到烷烃中是早期 开发新型高能材料的首选策略. Litvinov 等 ${ }^{[87]}$ 以 $\mathrm{F}_{2} \mathrm{NO}-$ $\mathrm{SO}_{2} \mathrm{~F}$ 为二氟氨基试剂, 开展了二硝甲基烷烃化合物的 二氟氨基化研究, 首次合成了系列具有二氟氨基二硝甲 基结构的新型氧化剂二氟氨基三硝基甲烷 $\left[\left(\mathrm{NO}_{2}\right)_{3}\right.$ $\left.\mathrm{CNF}_{2}\right](\mathbf{3 5}) 、 1$-二氟氨基-1,1,2,2,2-五硝基乙烷 $\left[\left(\mathrm{NO}_{2}\right)_{3} \mathrm{C}\right.$ $\left.\mathrm{C}\left(\mathrm{NO}_{2}\right)_{2} \mathrm{NF}_{2}\right]$ (36)、氟代二氟氨基二硝基甲烷 $\left[\left(\mathrm{NO}_{2}\right)_{2} \mathrm{C}-\right.$ $\mathrm{F}\left(\mathrm{NF}_{2}\right)$ ] (37)、1,3-双 (二氟氨基)-1,1,3,3-四硝基丙烷 $\left.\left[\mathrm{CH}_{2} \mathrm{C}_{\left(\mathrm{NO}_{2}\right.}\right)_{2} \mathrm{NF}_{2}\right](\mathbf{3 8}) 、 1,5$-双 (二氟氨基化)-1,1,3,5,5-五 硝基-3-氮杂戊烷化合物 $\left\{\left[\mathrm{NF}_{2} \mathrm{C}\left(\mathrm{NO}_{2}\right)_{2} \mathrm{CH}_{2}\right]_{2} \mathrm{NNO}_{2}\right\}$ (39). 据 Shreeve 等 ${ }^{[86]}$ 引述, 化合物 39 密度达到 $2.04 \mathrm{~g} / \mathrm{cm}^{3}$, 这 在非环含能化合物中是非常高的. 二氟氨基二硝甲基的 成功构建为新型高能基团开启了新的方向, 推动了后续 二氟氨基二硝甲基化合物开发.

Fokin 等 ${ }^{[68]}$ 开展了反应底物结构对二硝甲基烷烃阴 离子二氟氨基化收率的影响研究. 以 $\mathrm{F}_{2} \mathrm{NOSO}_{2} \mathrm{~F}$ 为二氟 氨基化试剂, 分别完成了甲基/乙基羧酸酯二硝甲基锂 盐、钠盐和钾盐在乙腈中的二氟氨基化反应(Scheme 16), 发现阳离子种类对反应收率影响较大, 其中锂盐 收率最差, 未有目标产物生成, 钠盐收率最高, 收率均 超过了 $50 \%$. 研究还发现钾盐化合物更易引发 $\mathrm{F}_{2} \mathrm{NO}-$ $\mathrm{SO}_{2} \mathrm{~F}$ 发生歧化反应, 生成 $\mathrm{FNO}$ 和 $\mathrm{SO}_{2} \mathrm{~F}_{2}$, 不利于反应进 行. 基于上述结果, 作者尝试使用该方法对 1,1,3,3-四硝 基丙烷二钠盐进行二氟氨基化, 但却导致了底物的剧烈 分解. 由此表明, 二硝甲基邻位基团(如羧酸酯等)对该 反应也有一定的影响.

为了能够实现 1,1,3,3-四硝基丙烷二钠盐等化合物 的二氟氨基化, Fokin 等系统地开展了溶剂极性对二硝 甲基钠盐、锂盐化合物的二氟氨基化的影响规律研究.

$$
\begin{aligned}
& \begin{array}{ccc}
\mathrm{XC}\left(\mathrm{NO}_{2}\right)_{2} \mathrm{COOR} & \stackrel{\mathrm{F}_{2} \mathrm{NOSO}_{2} \mathrm{~F}}{\underset{\mathrm{CH}_{3} \mathrm{CN}}{\longrightarrow}} & \mathrm{NF}_{2} \mathrm{C}\left(\mathrm{NO}_{2}\right)_{2} \mathrm{COOR} \\
-20 \text { to } 20^{\circ} \mathrm{C} & \mathbf{4 0} \sim \mathbf{4 1}
\end{array} \\
& \mathrm{X}=\mathrm{Na}, \mathrm{Li}, \text { or } \mathrm{K} ; \mathrm{R}=\mathrm{Me}(\mathbf{4 0}), \mathrm{Et}(41)
\end{aligned}
$$

图式 16 偕二硝基乙酸酯阴离子二氟氨基化反应 Scheme 16 Difuoroamination of alkoxycarbonyl dinitromethanides

由于该反应在大极性溶剂 $\mathrm{MeCN}(\varepsilon=37.5)$ 和小极性溶 剂 $\mathrm{CH}_{2} \mathrm{Cl}_{2}(\varepsilon=8.9)$ 中都未成功, 因此该课题组尝试极性 适中的 $\mathrm{Me}_{2} \mathrm{CO}(\varepsilon=20.7) 、 \mathrm{MeCO}_{2} \mathrm{Et}(\varepsilon=6.0)$ 、二甘醇二 甲醚 $(\varepsilon=7.5)$ 以及混合溶剂环丁砜 $-\mathrm{CH}_{2} \mathrm{Cl}_{2}(V / V=\mathrm{I}: 2$, $\varepsilon=24.7) 、 \mathrm{MeCN}^{-C_{2}} \mathrm{Cl}_{2}(V / V=1: 1 、 1: 2 、 1: 3, \varepsilon=$ $26.1 、 21.2$ 和 19.9)作为反应溶剂. 实验发现, 反应温度 为 $-20 \sim-40{ }^{\circ} \mathrm{C}$ 时，在二甘醇二甲醚中会导致反应底 物剧烈分解; 其他溶剂中随反应的进行, $\mathrm{F}_{2} \mathrm{NOSO}_{2} \mathrm{~F}$ 都 会迅速被消耗，底物二硝甲盐也很快溶解，但只有在体 积比为 $1: 2$ 和 $1: 3$ 的 $\mathrm{MeCN}^{-} \mathrm{CH}_{2} \mathrm{Cl}_{2}$ 混合溶剂中分离 获得相应的二氟氨基二硝甲基化合物(Scheme 17). 产 物的 43a, 43d, 43e 收率相对较好, 分别为 $65 \%, 41 \%$ 和 25\%.1,2-双(二氟氨基)-1,1,2,2-四硝基乙烷(43c) 分子结

\begin{tabular}{|c|c|c|c|}
\hline \multicolumn{2}{|c|}{$\begin{array}{l}\mathrm{XC}\left(\mathrm{NO}_{2}\right)_{2} \mathrm{R} \\
42 \mathrm{a} \sim 42 \mathrm{e}\end{array}$} & $\frac{\mathrm{F}_{2} \mathrm{NOSO}_{2} \mathrm{~F}}{-30 \text { to } 5^{\circ} \mathrm{C}}$ & $\begin{array}{c}\rightarrow \mathrm{NF}_{2} \mathrm{C}\left(\mathrm{NO}_{2}\right)_{2} \mathrm{R}^{\prime} \\
\quad 43 \mathrm{a} \sim 43 \mathrm{e}\end{array}$ \\
\hline & $\mathrm{R}$ & & $\mathrm{R}^{\prime}$ \\
\hline a & $\mathrm{Br}$ & & $\mathrm{Br}$ \\
\hline b & $\mathrm{CH}_{2} \mathrm{OH}$ & & $\mathrm{CH}_{2} \mathrm{OH}$ \\
\hline c & $\mathrm{C}\left(\mathrm{NO}_{2}\right)_{2} \mathrm{X}$ & & $\mathrm{C}\left(\mathrm{NO}_{2}\right)_{2} \mathrm{NF}_{2}$ \\
\hline d & $\mathrm{CH}_{2} \mathrm{C}\left(\mathrm{NO}_{2}\right)$ & & $\mathrm{CH}_{2} \mathrm{C}\left(\mathrm{NO}_{2}\right)_{2} \mathrm{NF}_{2}$ \\
\hline e & $\mathrm{CH}_{2} \mathrm{C}\left(\mathrm{NO}_{2}\right.$ & )$_{2} \mathrm{CH}_{2} \mathrm{OH}$ & $\mathrm{CH}_{2} \mathrm{C}\left(\mathrm{NO}_{2}\right)_{2} \mathrm{CH}_{2} \mathrm{OH}$ \\
\hline
\end{tabular}
构空间上高度拥挤，导致其收率最低，仅为 $5 \% \sim 7 \%$.

图式 17 偕二硝基烷烃阴离子二氟氨基化反应 Scheme 17 Difuoroamination of alkyl dinitromethanides

随后，该课题组以 2,2,4,4-四硝基戊烷-1,5-二醇(44) 为原料, 在氧化镁的水溶液中使用 $\mathrm{F}_{2} \mathrm{NOSO}_{2} \mathrm{~F}$, 完成了 4-二氟氨基-2,2,4,4-四硝基正丁醇(45)的合成，反应过程 中伴有副产物 1,3 ,-双 (二氟氨基)-1,1,3,3-四硝基丙烷 (43d)的生成(Eq. 1).

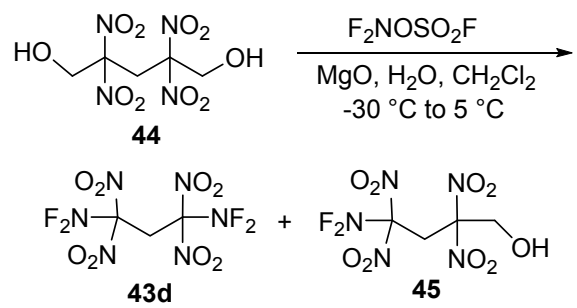

基于三硝甲基化合物与亲核试剂普遍具有较好的 
反应性, Khisamutdinov 等 ${ }^{[88]}$ 以无水 $N, N$-二甲基甲酰胺 (DMF)为溶剂, 开展了二氟氨基三硝基甲烷(46)与亲核 试剂 KF、CsF、LiBr 反应研究(Scheme 18). 结果发现, 与 多硝基甲烷类似，化合物 $\mathbf{4 6}$ 中一个硝基被氟取代，生成 了二氟氨基二硝基氟代甲烷(47), 反应收率与亲核试剂 阳离子种类有关, $\mathrm{KF}$ 作亲核试剂时收率仅为 $9 \%$, 而采 用 $\mathrm{CsF}$ 时收率可达 $40 \%$. 反应过程中生成的二氧化氮阴 离子 $\left(\mathrm{NO}_{2}{ }^{-}\right)$会与原料 46 发生反应, 生成二氟氨基二硝甲 基阴离子 48, 该阴离子在 DMF 中会进一步分解成氮氧 化物，因此导致总体收率较低.

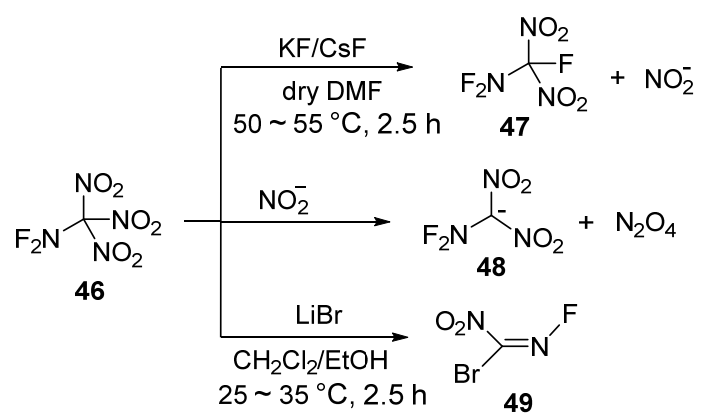

图式 18 化合物 46 与亲核试剂的反应

Scheme 18 Reactions of $\mathbf{4 6}$ with nucleophiles

当该研究组尝试将二氟氨基三硝基甲烷与 $\mathrm{LiBr}$ 反 应, 希望溴阴离子像氟离子一样取代一个硝基生成二氟 氨基二硝基溴代甲烷(50)时, 结果却生成了 $N$-氟代溴代 硝基甲烷(49), 推测的两条可能的反应过程如 Scheme 19 所示. 首先, 原料 46 与溴阴离子发生亲核取代反应, 生成中间体 50, 与溴代三硝基甲烷一样, 50 在溴的诱导 下发生氧化还原反应生成不稳定的阴离子化合物 $\mathbf{4 8}$, 随后发生氟消除反应得到 51 , 最后再与溴阴离子发生 取代生成 49. 另外一条可能的途径是, 46 与溴阴离子连
续发生两次取代反应生成 $\mathbf{5 2}$ ，与四硝基甲烷生成二溴 二硝基甲烷一样，通过单电子转移得到的自由基阴离子 化合物 53 进一步分解成硝基甲烷阴离子化合物 54, 最 后氟负离子离去转变成目标物 49.

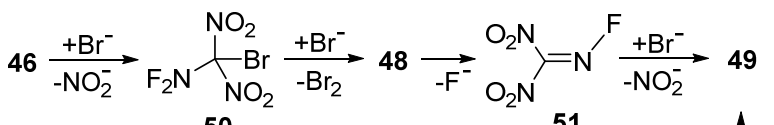

$$
\begin{aligned}
& +\mathrm{Br}^{-} \downarrow-\mathrm{NO}_{2}^{-} \quad 51 \quad-\mathrm{F}^{-} \uparrow
\end{aligned}
$$

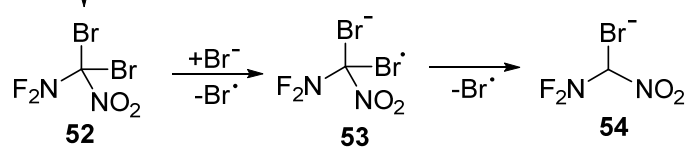

图式 19 化合物 46 与溴阴离子的反应机理

Scheme 19 Reaction mechanism of $\mathbf{4 6}$ with bromide anion

\section{2 二氟氨基二硝甲基取代氮杂芳环化合物}

氮杂芳环含能化合物是近年研究的热点, 利用氮杂 芳环反应位点向其引入硝基、硝胺基、硝酸酯、多硝甲 基等含能基团, 结合氮杂芳环 $\mathrm{N}-\mathrm{H}$ 键的酸性与各种富 氮碱匹配，能够实现含能材料性能的有效调节，获得性 能各异的新型含能材料. 高能基团二氟氨基二硝甲基与 氮杂芳环结合是探索高能量密度化合物的必然选择.

二氟氨基二硝基乙腈 $(\mathbf{5 5})^{[89]}$ 是合成二氟氨基化合物 的重要中间体，其结构稳定且氰反应活性高，为构筑二 氟氨基二硝甲基含能化合物提供基础. Fokin 等 ${ }^{[90]}$ 利用 55 在干燥氯化氢乙醇溶液中发生氰基聚合反应，生成 1,3,5-三(二氟氨基二硝甲基)均三嗪(56) (Scheme 20), 但 该反应可重复性较差，收率介于 $1 \% \sim 30 \%$ 之间. 利用氰 基与叠氮化钠环化反应得到其四唑钠盐中间体，使用 $\mathrm{F}_{2} \mathrm{NOSO}_{2} \mathrm{~F}$ 进一步对钠盐二氟氨基化, 获得了 $N-\mathrm{NF}_{2}$

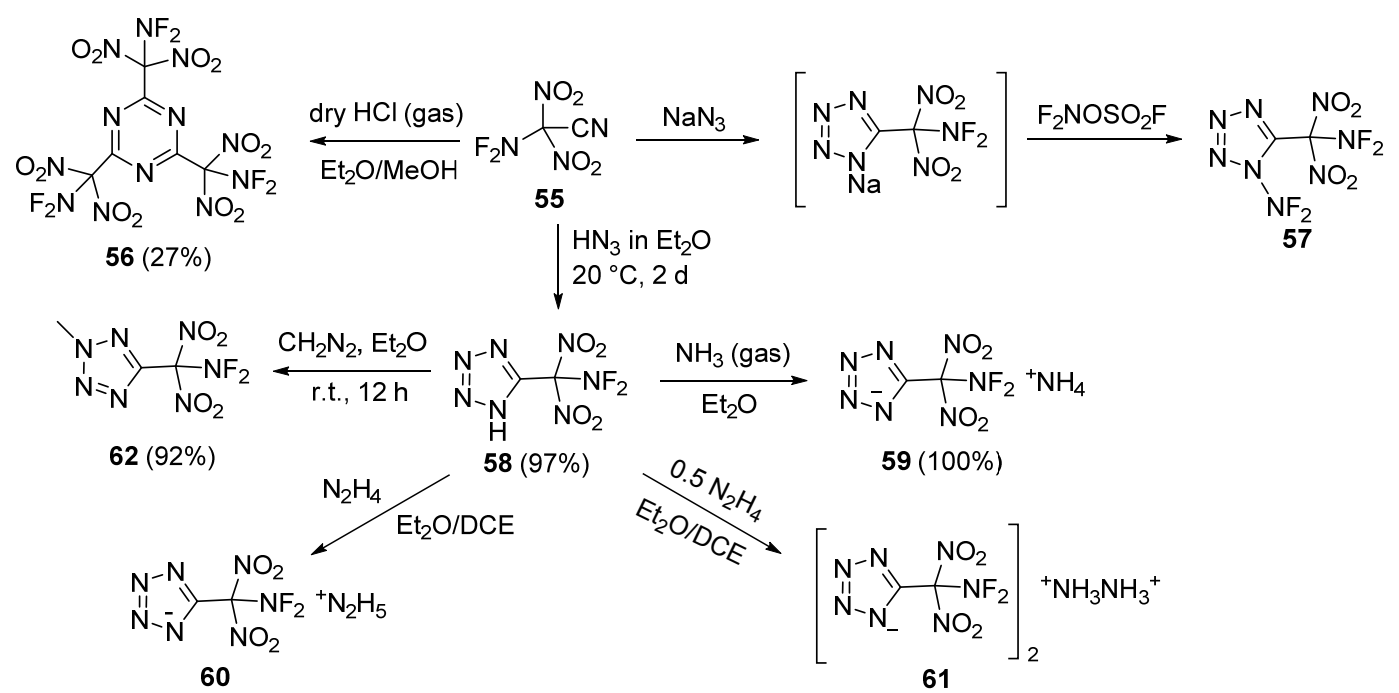

图式 20 二氟氨基二硝甲基取代的三嗪和四唑化合物的合成

Scheme 20 Synthesis of (difluoroamino)dinitromethyl-substituted triazine and tetrazole 
四坐化合物(57), 但该化合物过于敏感, 并未完成其结 构鉴定. 随后, 该研究团队又将 $\mathbf{5 5}$ 与 $\mathrm{HN}_{3}$ 在低温乙醚溶 剂中合成了 58, 收率高达 $97 \%$. 化合物 54 在室温下存 放二周后会缓慢分解, 但能够稳定存在于醚溶液中. 利 用四唑酸性得到了其铵盐 59 和肼盐 $60 \sim 61$, 与重氮甲 烷反应生成了 2-甲基-5-二氟氨基二硝甲基四唑(62)和 1甲基-5-二氟氨基二硝甲基四唑, 二者量约为 $16 ： 1$. 铵 盐 59 和甲基化产物 62 非常稳定, 铵盐熔点为 131 $132{ }^{\circ} \mathrm{C}$, 铵根离子与二氟氨基二硝甲基之间产生的分子 间氢键有助于结构的稳定.

2015 年, Dalinger 等 ${ }^{[69]}$ 开展了向吡唑 $N$-二硝甲基的 二氟氨化研究, 以 3,5-硝基-1-二硝甲基吡唑钾盐(63)为 原料, 在 $-15{ }^{\circ} \mathrm{C}$ 的乙腈溶液中, 利用 $\mathrm{F}_{2} \mathrm{NOSO}_{2} \mathrm{~F}$ 对其二 氟氨基化, 首次将二氟氨基二硝甲基引入到吡唑环中, 收率达 75\% (Eq. 2). 化合物 64 实测密度为 $1.92 \mathrm{~g} / \mathrm{cm}^{3}$, 生成焓为 $193 \mathrm{~kJ} / \mathrm{mol}$, 分解温度为 $113{ }^{\circ} \mathrm{C}$, 理论爆速和 爆压为 $8721 \mathrm{~m} / \mathrm{s}$ 和 $36.3 \mathrm{Gpa}$, 可作为潜在的高能氧化剂 使用.
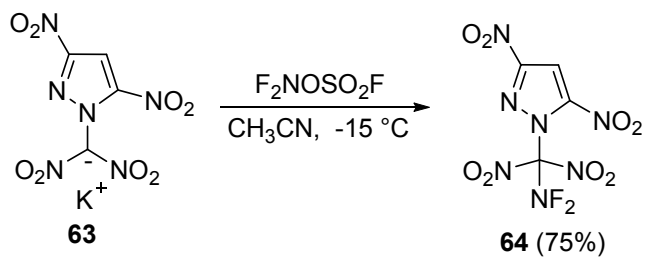

2017 年, Semenov 等 ${ }^{[70]}$ 采用同样的方法, 开展了两 个异构体 2,2'-双 (二硝甲基)-5,5'-二硝基联(1,2,4-三唑)和 2,2'-双 (二硝甲基)-5, $5^{\prime}$-二硝基联(1,2,3-三唑)的二氟氨基 化研究, 使用其钠盐在更低的反应温度下 $(-30 \sim-$ $40{ }^{\circ} \mathrm{C}$ ) 进行, 收率分别为 70\%和 50\%, 如 Scheme 21 所 示. 单晶结构显示, $2,2^{\prime}$-双 (二氟氨基二硝甲基)-5,5'-二硝 基联(1,2,4-三唑)(68)结构中两个三唑环共平面, 晶体密
度为 $1.896 \mathrm{~g} / \mathrm{cm}^{3}$. 2,2'-双 (二氟氨基二硝甲基)-5,5'二二硝 基联(1,2,3-三唑)(72)晶体结构显示两个硝基在分子同一 侧，有一定的空间位阻效应，导致两个三唑环存在一定 的夹角，其晶体密度为 $1.936 \mathrm{~g} / \mathrm{cm}^{3}$, 高于其异构体 $\mathbf{5 4}$, 表明取代基立体效应对含能分子堆积密度影响较大.

为了进一步提高二氟氨基二硝甲基吡唑化合物的 能量, 更好探究其与氟二硝甲基、三硝甲基类似物结构 和性能差异, Dalinger 等 ${ }^{\left[{ }^{71]}\right.}$ 于 2018 年开展了二氟氨基二 硝甲基、氟二硝甲基和三硝甲基取代的四唑联吡唑化合 物的合成、结构与性能对比研究, 如 Scheme 22 所示. 以 四唑联吡唑(73)为母体，通过硝化和取代两步反应得到 关键中间体 75, 使用 $100 \% \mathrm{HNO}_{3} / 100 \% \mathrm{H}_{2} \mathrm{SO}_{4}$ 对 75 再 次硝化获得二硝甲基钾盐化合物 76 和三硝甲基产物 77, 利用 Selectfluor 和 $\mathrm{F}_{2} \mathrm{NOSO}_{2} \mathrm{~F}$ 分别对钾盐 76 氟化和二 氟氨基化得到其氟二硝甲基产物 78 和二氟氨基二硝甲 基产物 79. 通过化合物 77、78 和 79 单晶结构分析发现, 四唑和硝基处于吡唑环的邻位，存在一定位阻效应，导 致四唑环和吡唑环具有较大的二面角 $\left(40^{\circ} \sim 60^{\circ}\right)$. 在一 $173 \mathrm{~K}$ 下, 77、78 和 79 晶体密度分别为 $1.857,1.873$ 和 $1.972 \mathrm{~g} / \mathrm{cm}^{3}$, 证实了二氟氨基对含能分子密度的显著贡 献. 通过对晶体中分子间弱作用力分析发现, 氟原子参 与分子间范德华作用的程度对化合物密度有直接影响, 79 分子中两个氟原子与其他分子间硝基氧及四唑氮形 成了数量可观的 $\mathrm{F} \cdots \mathrm{O}$ 和 $\mathrm{F} \cdots \mathrm{N}$ 作用, 有效地拉近了分 子间距离, 从分子层面解释了 79 密度高的原因.

表 2 列举了 $77 、 78$ 和 79 的热分解温度、密度、爆 速等性能. 由表 2 可知, 四唑的引入使得三个化合物的 生成焓普遍较高, 但氟二硝甲基、三硝甲基和二氟氨基 二硝甲基对生成焓贡献差别较大，其中氟二硝甲基化合 物 78 生成焓最小, 化合物 77 和 79 生成焓基本相当. 让

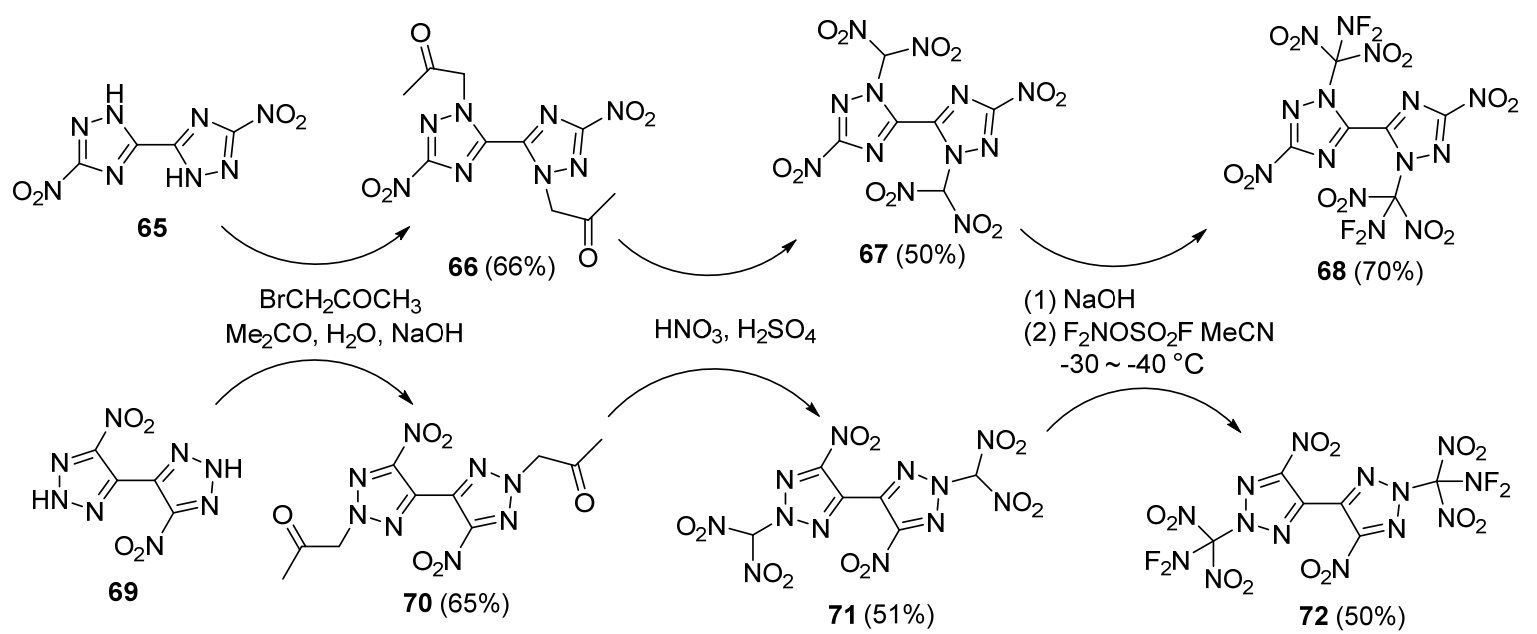

图式 21 二氟氨基二硝甲基三唑化合物的合成

Scheme 21 Synthesis of (difluoroamino)dinitromethyl-substituted triazoles 


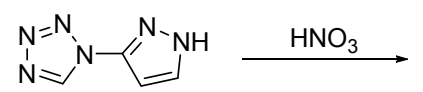

73
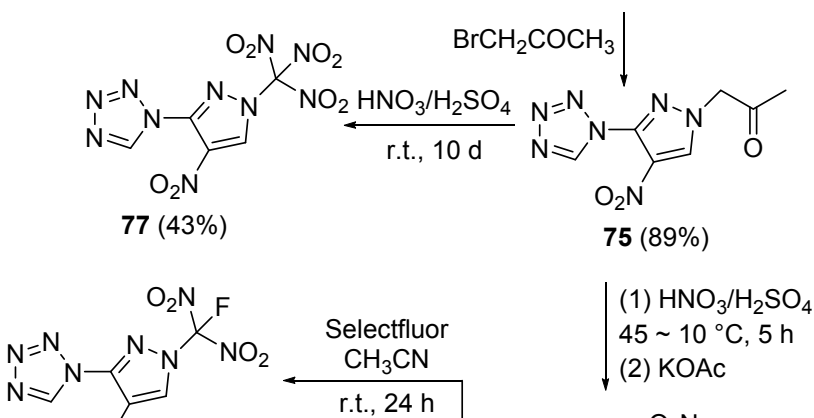

$\mathrm{O}_{2} \mathrm{~N}$

$78(82 \%)$

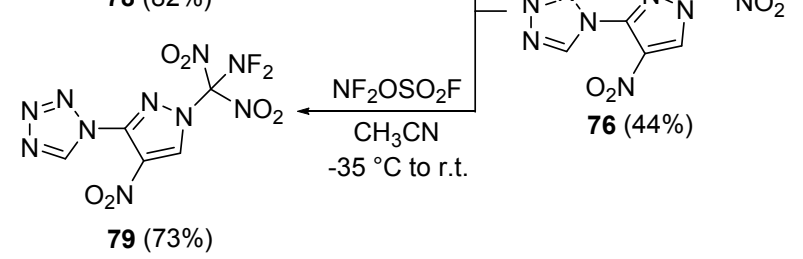

图式 22 多硝甲基吡唑化合物的合成

Scheme 22 Synthesis of polynitromethyl-substituted pyrazole

表 2 化合物 77、78 和 79 性能对比

Table 2 Comparison of properties of compounds 77, 78 and 79

\begin{tabular}{|c|c|c|c|c|c|c|}
\hline Substrate & $T_{\mathrm{dec}} /{ }^{\circ} \mathrm{C}$ & $\begin{array}{c}\Delta H_{\mathrm{f}} f \\
\left(\mathrm{~kJ} \cdot \mathrm{mol}^{-1}\right)\end{array}$ & $\begin{array}{c}\rho / \\
\left(\mathrm{g} \bullet \mathrm{cm}^{-3}\right)\end{array}$ & $\begin{array}{c}V_{\text {det }} \\
\left(\mathrm{m} \cdot \mathrm{s}^{-1}\right) \\
\end{array}$ & $\begin{array}{l}P_{\mathrm{CJ}} / \\
\mathrm{GPa} \\
\end{array}$ & $I_{\mathrm{sp}} / \mathrm{s}$ \\
\hline 77 & 127 & 602 & 1.79 & 8860 & 34 & 273 \\
\hline 78 & 111 & 386 & 1.81 & 8470 & 31 & 262 \\
\hline 79 & 138 & 589 & 1.91 & 8990 & 36 & 282 \\
\hline $\mathrm{NG}^{[71]}$ & 125 & -351.5 & 1.60 & 7630 & 22 & 259 \\
\hline $\operatorname{PETN}^{[71]}$ & 181 & -538 & 1.77 & 8330 & 30 & 263 \\
\hline $\mathrm{TNT}^{[71]}$ & 295 & -67 & 1.65 & 6663 & 21 & 210 \\
\hline $\mathrm{RDX}^{[71]}$ & 204 & 67 & 1.82 & 8850 & 35 & 266 \\
\hline
\end{tabular}

人意外的是, 二氟氨基化合物 79 分解温度高于 77 和 78 , 表明二氟氨基二硝甲基具有良好的结构稳定性. 高的密 度和生成焓赋予了化合物 79 优异爆轰性能, 其爆速接 近 $9000 \mathrm{~m} / \mathrm{s}$ ，爆压达 $36 \mathrm{GPa}$ ，优于相应的氟二硝甲基化 合物 78 和三硝甲基化合物 77. 化合物 79 更大的优势在 于比冲，其理论比冲 $\left(I_{\mathrm{sp}}=282 \mathrm{~s}\right)$ 显著高于季戊四醇四硝 酸酯(PETN, 263s)、TNT (210s) 和 RDX (266 s)等化合物. 由此可以看出, 基于 $\mathrm{N}-\mathrm{F}$ 键的高能化合物在密度、爆 速、爆压和比冲方面优势明显, 佐证了该类化合物潜在 的开发前景.

\section{4 偕二氟氨基含能化合物}

为了避免具有 $\alpha-\mathrm{H}$ 结构的二氟氨基化合物自身发 生 $\mathrm{HF}$ 消除反应, 以无 $\alpha-\mathrm{H}$ 结构的偕二氟氨基化合物逐 渐成为二氟氨基化合物研究热点. 羰基等不饱和官能团 与二氟氨基化试剂 $\mathrm{HNF}_{2} 、 \mathrm{NF}_{2} \mathrm{SO}_{2} \mathrm{~F}$ 等发生亲核加成反
应，是获得偕二氟氨基含能化合物主要途径.

\section{1 链状偕二氟氨基含能化合物}

链状偕二氟氨基含能化合物是最早开发的一类偕 二氟氨基含能化合物，并证实了无 $\alpha-\mathrm{H}$ 结构的偕二氟氨 基化合物的结构稳定性，促进了后续环状偕二氟氨基含 能化合物的发展. Baum ${ }^{[58]}$ 以不同取代基的甲基酮链状 化合物为底物, 在强酸条件下与 $\mathrm{HNF}_{2}$ 发生亲核加成反 应，获得了多种结构稳定的偕二氟氨基链状化合物，如 Scheme 23 所示. 二氟氨基化反应收率受反应底物结构、 反应体系酸性强度等影响较大. 实验发现, 具有吸电子 取代基的化合物需要强化反应条件，如加大 $\mathrm{HNF}_{2}$ 浓度、 更高的反应温度、更强的酸性介质(如发烟硫酸)等。以 5,5,5-三硝基-2-戊酮(80e)为例，使用浓硫酸在回流温度 反应 $4 \mathrm{~h}$ 时收率为零; 当 $80 \mathrm{e}$ 浓度为 $4 \mathrm{~mL} / \mathrm{mmol}$, 使用 $100 \%$ 硫酸、 $\mathrm{HNF}_{2}$ 过量 8 倍时, 室温下反应 $40 \mathrm{~h}$ 的收率 为 $53 \%$. 当底物浓度进一步降低至 $0.7 \mathrm{~mL} / \mathrm{mmol}$, 使用 $20 \%$ 发烟硫酸、3 倍 $\mathrm{HNF}_{2}$ ，室温下 $2 \mathrm{~h}$ 的收率即可高达 $99.5 \%$. 由此可见，该类型反应收率受反应条件强弱和 底物结构影响较大.

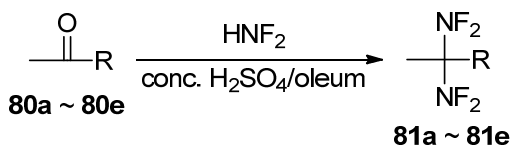

$$
\begin{aligned}
& \mathrm{R}=\mathrm{CH}_{3}(\mathbf{a}), \mathrm{CH}_{2} \mathrm{Cl} \text { (b), }\left(\mathrm{CH}_{2}\right)_{3} \mathrm{NO}_{2}(\mathbf{c}),\left(\mathrm{CH}_{2}\right)_{2} \mathrm{C}\left(\mathrm{NO}_{2}\right)_{2} \mathrm{CH}_{3}(\mathbf{d}) \text {, } \\
& \left(\mathrm{CH}_{2}\right)_{2} \mathrm{C}\left(\mathrm{NO}_{2}\right)_{3}(\mathbf{e})
\end{aligned}
$$

图式 23 链状酮化合物二氟氨基化反应

Scheme 23 Difluoramination of chain ketones

Coon 等 ${ }^{[63]}$ 以 2-丁烯-1,4-二醇为原料, 通过氧化、加 成、酯化及二氟氨基化等反应，报道了一种含偕二氟氨 基和氟二硝甲基的高能增塑剂 2,2-双 (二氟氨基)-5-氟5,5-二硝基-1-戊醇 (86) (Scheme 24). 化合物 86 外观呈浅 黄色, 熔点为 $14{ }^{\circ} \mathrm{C}$, 沸点为 $79 \sim 80{ }^{\circ} \mathrm{C}$, 该化合物分子 结构稳定, 且氟含量较高, 将其作增塑剂或氧化剂使用,
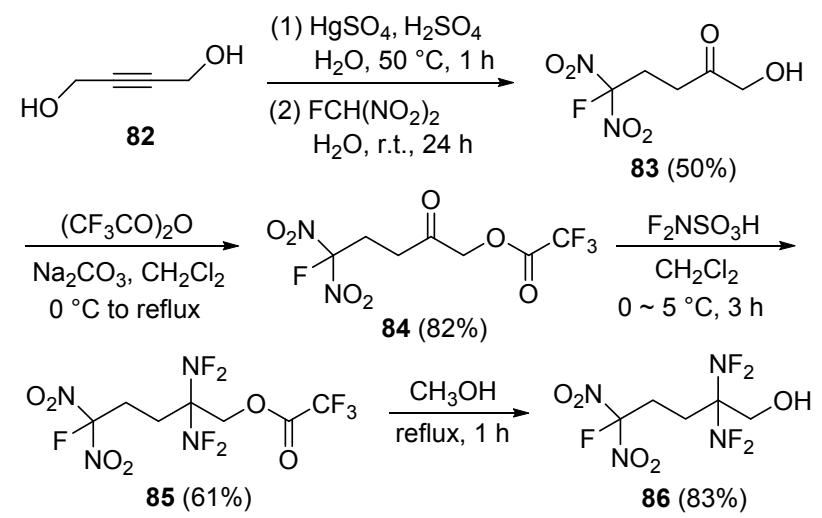

图式 24 化合物 86 的合成 Scheme 24 Synthesis of 86 
能够显著提高双基推进剂的比冲和燃速. Baum 等 ${ }^{[64]}$ 利 用同样的方法，对 1,3-双(2,2,2-氟二硝基乙氧基)丙酮进 行二氟氨基化, 获得 1,3-双(2,2,2-氟二硝基乙氧基)-2,2偕二氟氨基丙烷, 分子能量和氧含量得到进一步提升.

利用叠氮基高生成焓 $(356 \mathrm{~kJ} / \mathrm{mol})$ 的特点, Flanagan 等 ${ }^{[65}$ 设计合成了一种具有两个叠氮基和偕二氟氨基结 构的化合物 (89). 以 1,7-二氯-4-庚酮为原料(87), 通过二 氟氨基化和叠氮负离子取代反应合成了 1,7 -二叠氮基4,4-偕二氟氨基庚烷 (89), 如 Scheme 25 所示. 为了进一 步考察其应用可行性, 国内黎明化工研究院张明权 等 ${ }^{[91]}$ 开展了合成工艺优化, 确定了二氟氨基化反应原 料配比, 将总收率提高到 $70 \%$, 并报道了化合物 89 起始 分解温度为 $195.2{ }^{\circ} \mathrm{C}$, 表明热稳定性良好. 为了降低其 感度, 张明权等采用复合降感剂, 将化合物 89 的撞击感 度由 $5.9 \mathrm{~cm}$ 改善至 $30 \mathrm{~cm}$ 以上, 摩擦感度由 $100 \%$ 降低 至 $10 \%$ 以下, 为化合物 89 的安全使用提供了保证.

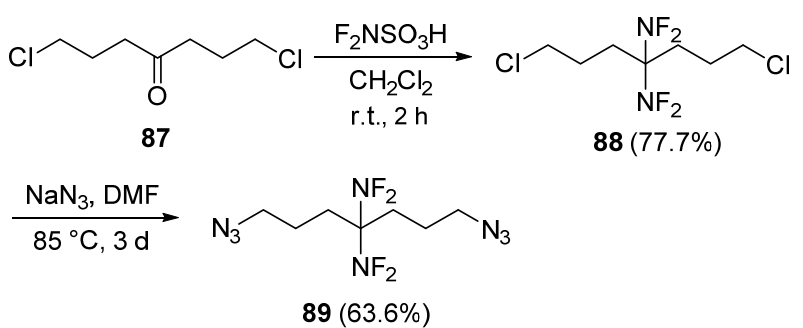

图式 25 化合物 89 的合成

Scheme 25 Synthesis of 89

Frankel 等 ${ }^{[92]}$ 以 3 -氟二硝乙氧基-1-氯-2-丙醇 $(90)$ 为 原料，通过氧化、二氟氨基化、取代等多步反应合成了 1- 叠氮-2,2-双(氟二氨基)-3-氟二硝乙氧基丙烷(93) (Scheme 26), 该化合物分子中同时含有偕二氟氨基、叠 氮基和氟二硝甲基, 因此具有高生成焓、富氧等特点, 在推进剂组分中可以作为含能增塑剂和高能氧化剂使 用.

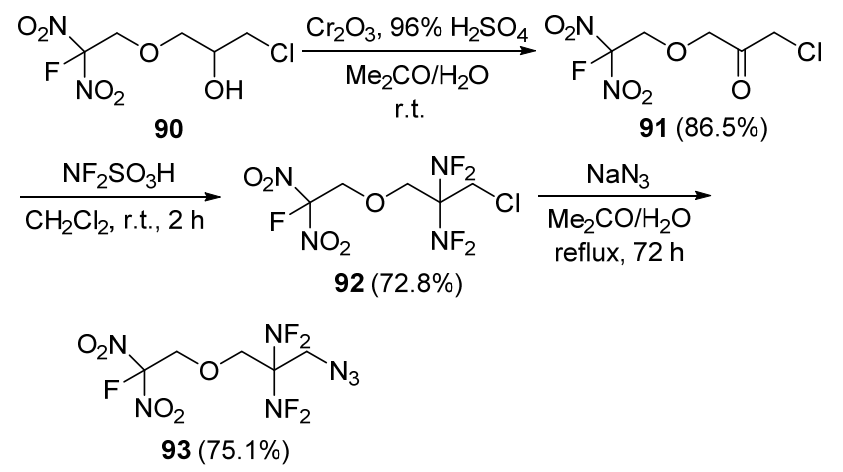

图式 26 化合物 93 的合成

Scheme 26 Synthesis of 93

为了开发合成步骤简单且能量水平进一步提高的
偕二氟氨基含能增速剂, Adolph 等 ${ }^{\left[{ }^{[3]}\right.}$ 以 1,5-二氯戊烷-3酮(94)为原料, 通过三步反应合成了 3,3 -双(二氟氨基)1,5-二硝酸酯基戊烷(99), 如 Scheme 27 所示. 该分子含 有二个硝酸酯基和一个偕二氟氨基, 密度为 $1.56 \mathrm{~g} / \mathrm{cm}^{3}$, 玻璃化转变温度为 $-87{ }^{\circ} \mathrm{C}$, 初始分解温度为 $186{ }^{\circ} \mathrm{C}$, 峰温为 $196{ }^{\circ} \mathrm{C}$, 生成热为 $-320.0 \mathrm{~kJ} / \mathrm{mol}$, 其理论比冲 可达 278.9 s，显著高于 1,2,4-丁三醇三硝酸酯(BTTN, $262.3 \mathrm{~s})$ 和 1,3-双(2,2,2-氟二硝基乙氧基)-2,2-偕二氟氨 基丙烷(266.5 s). 与其他偕氟二氨基增塑剂相比，其在 合成难易程度、热稳定性及能量水平等方面均具有明显 优势.
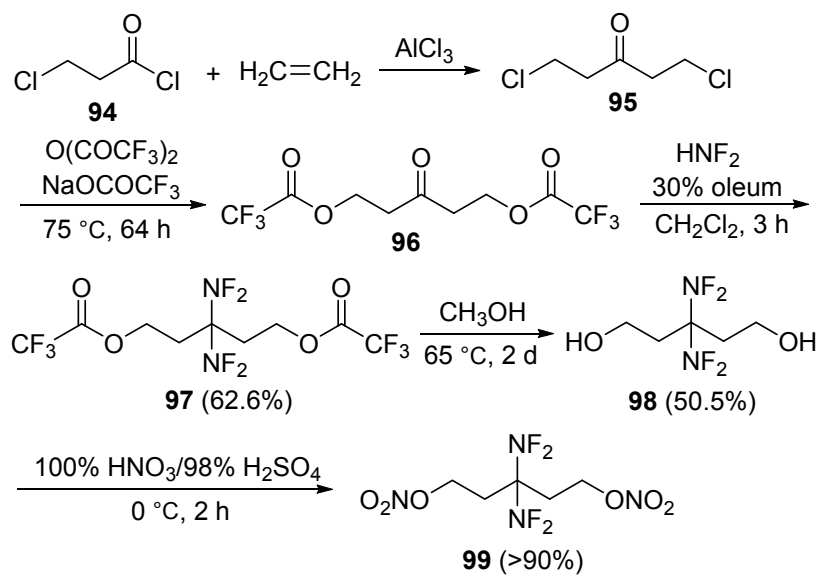

图式 27 化合物 99 的合成

Scheme 27 Synthesis of 99

\section{2 环状偕二氟氨基含能化合物}

以六元环和八元环为代表环状含能化合物(如 RDX 和 HMX)在含能材料中占有重要地位, 致密对称的环结 构赋予了此类化合物密度高、热稳定性好的特点. 近年, 以 HNFX 为代表的环状偕二氟氨基含能化合物展现出 了巨大的发展潜力.

HNFX 是类似 HMX 的八元环状偕二氟氨基硝胺化 合物，自其首次被设计出来便成为含能材料研究人员关 注的焦点. HNFX 的晶体密度为 $1.807 \mathrm{~g} / \mathrm{cm}^{3}$, 初始分解 温度为 $221{ }^{\circ} \mathrm{C}$, 峰温为 $231{ }^{\circ} \mathrm{C}$, 撞击感度与 CL-20 相 当, 摩擦感度和静电感度略高于 CL-20 和 PETN ${ }^{[29]}$. 目 前发现的 HNFX 晶体密度仅为 $1.807 \mathrm{~g} / \mathrm{cm}^{3}$, 这与 Ammon 等 ${ }^{[94]}$ 预估的 $2.027 \mathrm{~g} / \mathrm{cm}^{3}$ 差距较大. 但相关研究 人员普遍认为 HNFX 与 HMX、CL-20 一样存在多种晶 型，具有更高密度的晶型还有待进一步发现。Baum 等 ${ }^{[95]}$ 在一篇提交给美国海军研究局的报告中首次报道 了 HNFX 合成路线, 如 Scheme 28 所示. 在最后一步反 应中，由于中间体 102 分子中硝胺结构在强非硝酸条件 下易分解，致使最后一步反应收率仅为 $1 \%$ ，限制了进 一步性能及应用可行性研究. 

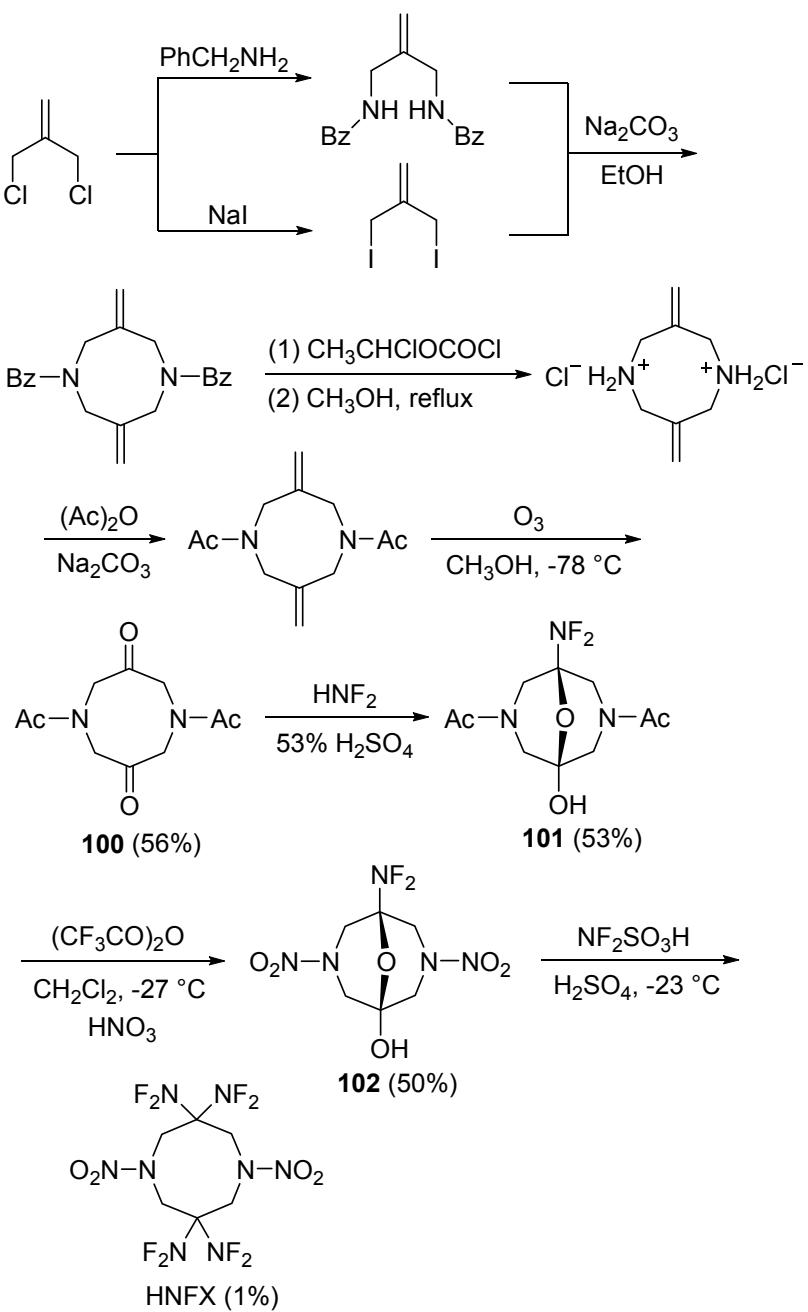

图式 28 HNFX 的合成

Scheme 28 Synthesis of HNFX

为了简化反应步骤并提高收率, Chapman 等 ${ }^{[96,97]}$ 研 究了 $N$-保护基种类对氮杂辛烷二酮二氟氨基化反应的 影响, 发现保护基的酸碱性对二氟氨基化和硝化反应具 有重要影响. 基于此, 该研究组以对硝基苯磺酰胺和环 氧氯丙烷为原料, 首先合成了对硝基苯磺酰基为保护基 中间体八氢-1,5-二(4-硝基苯磺酰基)-1,5-二氮杂辛烷3,7-二醇(103), 再利用斯文(Swern)氧化反应将其氧化成 二酮化合物 104, 该步收率为 $94 \%$. 随后通过优化反应 温度以及向二氟氨基化反应体系中加入惰性试剂 $\mathrm{CFCl}_{3}$, 将二氟氨基化收率提高至 $60 \%$. 最后, 利用篮选 的高效硝化体系 $\mathrm{HNO}_{3}-\mathrm{CF}_{3} \mathrm{SO}_{3} \mathrm{H}$, 将 HNFX 最后一步收 率由原来 $1 \%$ 大幅提高到 $65 \%$, 如 Scheme 29 所示.

尽管上述方法在小试合成 HNFX 时较为成功, 然而 放大后收率会显著下降. 为此, Chapman 等 ${ }^{[98,99]}$ 开发了 从二酮化合物 104 到 HNFX “一锅法” 合成工艺, 如 Scheme 30 所示. 通过向二氟氨基化体系中加入三氟磺 酸, 将氮上的对硝基苯磺酰基在三氟磺酸作用下脱去,

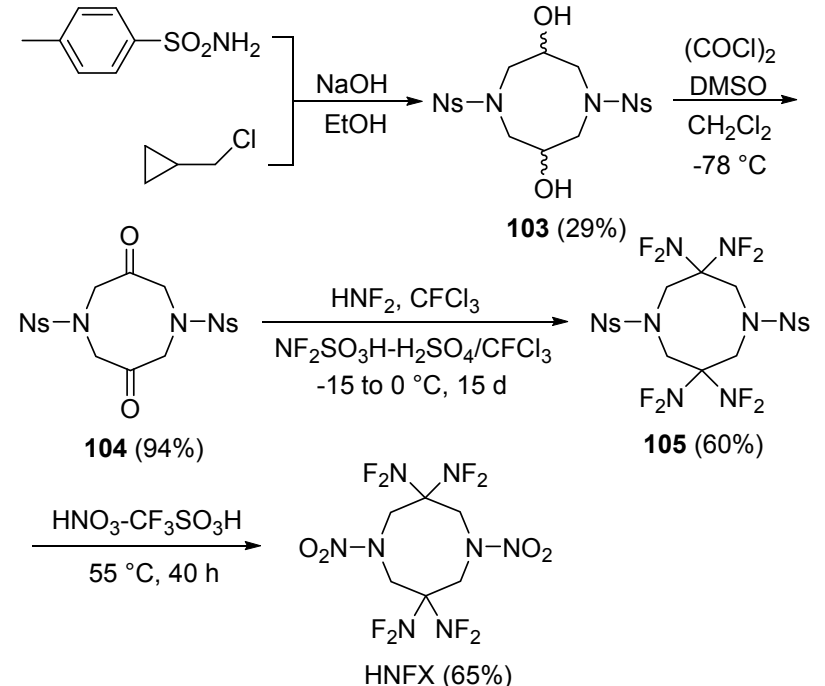

图式 29 HNFX 的合成路线改进

Scheme 29 Improved synthesis route of HNFX

成双氢取代的季铵正离子化合物 106 , 再向反应体系中 加入 $98 \% \sim 100 \%$ 硝酸进行硝化反应，一锅法合成 HNFX，将总收率提高到 75\%，中间体均不需要分离， 大大简化了实验步骤，为应用性能和基础应用研究提供 了基础.

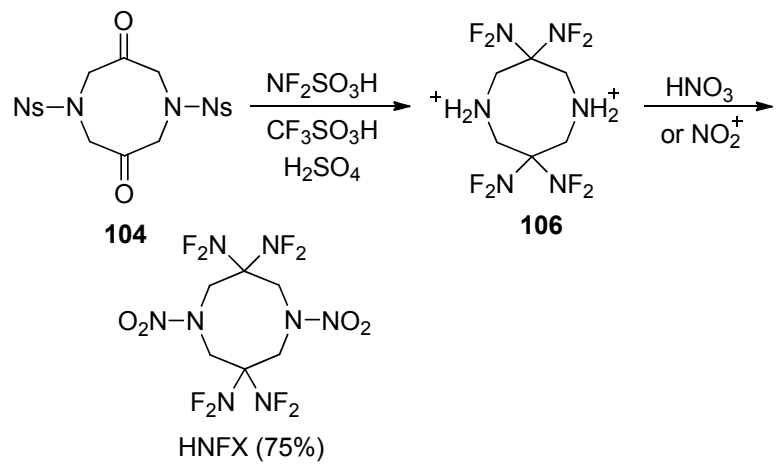

图式 $30 \mathrm{HNFX}$ 的一锅法合成

Scheme 30 One-pot synthesis process of HNFX

3,3 -双(二氟氨基)二八氢化-1,5,7,7-四硝基-1,5-二氮 杂辛烷(TNFX)结构与 HNFX 类似，将 HNFX 分子中一 个偕二氟氨基换成二硝甲基即得到 TNFX, 该化合物相 比 HNFX 氧平衡得到改善. 2001 年, Axenrod 等 ${ }^{[100]}$ 以 2羟基-1,3-丙二胺为原料, 经氨基保护、羟基氧化、环化 等构建了 1,5 -二氮杂辛烷母体化合物 $\mathbf{1 0 7}$, 随后利用与 HNFX 相似的硝化、二氟氨基化等多步反应合成了 TNFX，其中二氟氨基化步骤收率可达 $90 \%$ ，如 Scheme 31 所示. 研究发现, TNFX 存在多晶现象, 三方晶系的 晶体密度较低, 为 $1.712 \mathrm{~g} / \mathrm{cm}^{3}$, 而正交晶系的晶体密度 显著高于三方晶系，达 $1.902 \mathrm{~g} / \mathrm{cm}^{3}$, 这对寻找更高密度 的 HNFX 具有指导意义. 


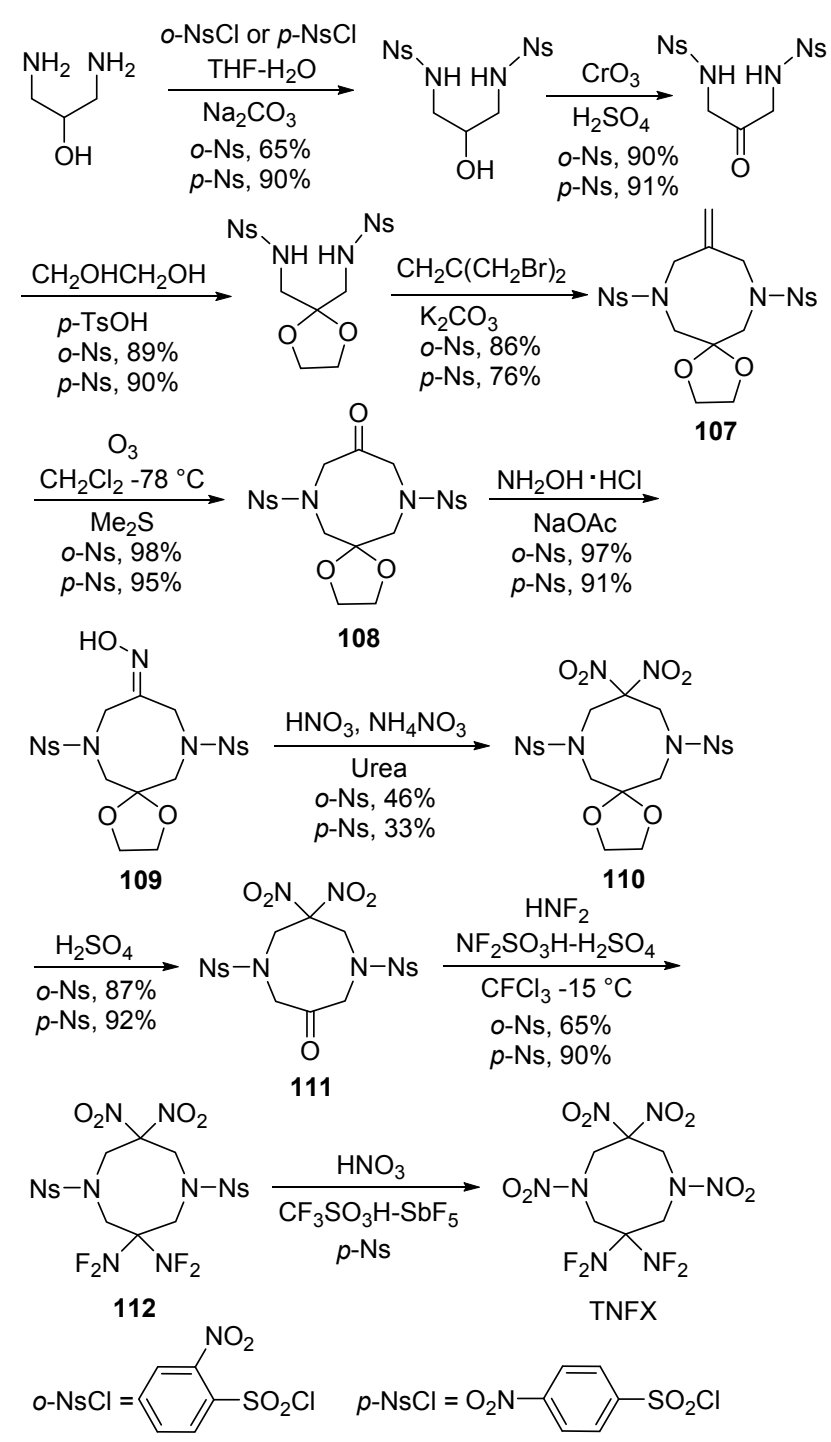

图式 31 TNFX 的合成

Scheme 31 Synthesis of TNFX

5,5-双 (二氟氨基)六氢-1,3-二硝基嘧啶 (RNFX) ${ }^{[101]}$ 是分子结构与 $\mathrm{RDX}$ 类似的六元环状偕二氟氨基硝胺化 合物, 是 Chapman 课题组继 HNFX之后合成的又一重要 标志性化合物. 合成六氢嘧啶酮是合成该化合物的关键 步骤之一, 与 HNFX 合成思路相似, 首先构建对硝基苯 磺酰基保护的六氢嘧啶酮 117. 公开的专利描述了两条 路线: 第一条路线是将对硝基苯磺酰胺先与甲醛作用, 生成硝基苯磺酰二胺化合物 $\mathbf{1 1 3}$, 收率仅为 $18 \%$, 随后 通过亲核取代环化和氧化反应得到 117, 收率为 $83 \%$ 。 第二条路线是使用 2-羟基丙二胺与甲醛缩合环化生成 着基取代的六氢嘧啶 115, 收率为 $88 \%$, 其与对硝基苯 磺酰胺的反应收率高达 $95 \%$, 但该专利并未报道后续氧 化生成 117 的具体步骤. 但将仲醇氧化成酮条件颇多, 如在合成 HNFX 时使用的斯文(Swern)氧化反应等, 作 者认为第二条合成路线无论从收率、成本等方面考虑均
更具优势. 同时，作者也设计另外两条合成路线，供读 者参考, 见 Scheme 32 所示.

参照合成 HNFX 和 TNFX 的二氟氨基化条件，中间 体 117 能够顺利转化成相应的偕二氟氨基中间体 118 . 当使用 $98 \%$ 硝酸对其硝解合成 RNFX 时, 六氢嘧啶环中 处在两个氮中间的亚甲基发生开环反应，生成中间体 119 , 在含有少量水的硝酸作用下, 119 进一步发生去苯 磺酰基化反应生成 121, 其可以立即与等量甲醛发生环 化反应, 得到目标化合物 RNFX. 该硝解过程与合成 HNFX 时硝化反应过程差别较大, 如 Scheme 33 所示. 尽管该化合物的合成报道己过多年，但其性能一直未见 后续报道. 该化合物分子结构较为完美，硝胺基和二氟 氨基在六元环中分布合理紧凑，并且合成步骤相对简 单，作者认为该化合物是目前最具潜力的氟氮含能化合 物之一.

除上述关注度较高的偕二氟氨基硝胺化合物外, Baum ${ }^{[58]}$ 以环状酮为底物, 利用 $\mathrm{HNF}_{2} / \mathrm{H}_{2} \mathrm{SO}_{4}$ 体系合成了 多种偕二氟氨基五元环和六元环烷烃化合物, 如表 3 所 示. 该方法需要在强酸条件下使用过量的 $\mathrm{HNF}_{2}$ 才能获 得较好的收率. 为了改善 $\mathrm{HNF}_{2}$ 使用带来的安全隐患, Prakash 等 ${ }^{[6]}$ 于 2002 报道了新型二氟氨基化试剂三苯基 二氟氨基甲烷，并用该试剂合成了多种环状二氟氨基化 化合物, 收率均能达到 $80 \%$ 以上, 为偕二氟氨基化合物 提供了一种安全、高效的合成方法.

表 3 环状偕二氟氨基烷烃衍生物

Table 3 Cyclic gem-bis(difluoramino) alkyl derivatives

Starting material

\section{5 其他氟氮含能化合物}

\section{1 新戊基二氟氨基化合物}

研究发现, 当二氟氨基与新戊基相连, 由于 $\left(\mathrm{CH}_{3}\right)_{3} \mathrm{CCH}_{2}$ 空间位阻效应，抑制了二氟氨基与 $\alpha-\mathrm{H}$ 发生 消除 $\mathrm{HF}$ 反应，是目前能够稳定存在具有 $\alpha-\mathrm{H}$ 结构的二 氟氨基化合物. 由于分子结构稳定性的大幅提升，因此 可以使用 $F_{2}$ 直接氟化新戊基胺来合成二氟氨基化合物, 该方法工艺简单, 有利于放大实验. Manser 等 ${ }^{\left[{ }^{[0]}\right.}$ 以 3 -氨 


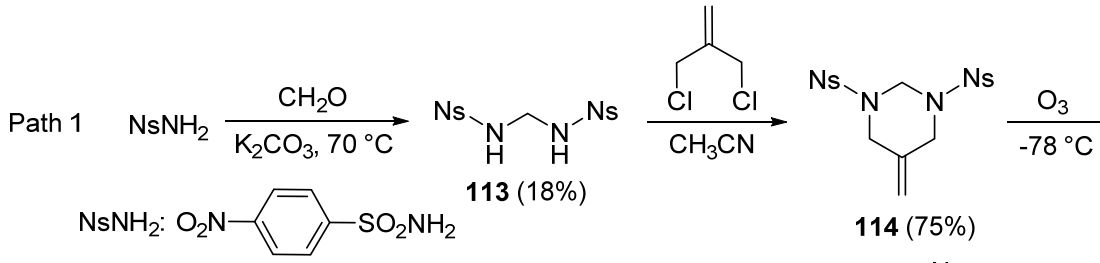

Path $2 \underbrace{\mathrm{NH}_{2}}_{\mathrm{OH}} \frac{\mathrm{NH}_{2}}{\text { r.t., 3d }}$

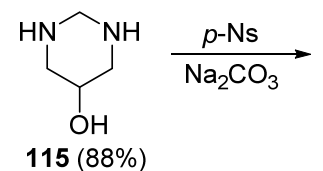

$\underbrace{\mathrm{Ns}}_{\mathrm{OH}}$ oxidation $\mathrm{OH}$

Path 3

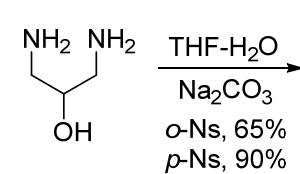

Ns、

$\underbrace{\mathrm{NH} \mathrm{HN}}_{\mathrm{OH}} \frac{\begin{array}{c}\mathrm{Ns} \\ 0-\mathrm{Ns}, 90 \% \\ p-\mathrm{Ns}, 91 \%\end{array}}{\mathrm{CrO}_{3}}$

Ns

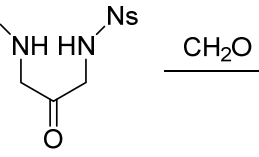

Ns ${ } \widehat{N}_{\mathrm{N}^{-}}-\mathrm{Ns}$

$\underbrace{\mathrm{OH}}_{\mathrm{OH}} \frac{\substack{\mathrm{O}-\mathrm{Ns}, 65 \% \\ \mathrm{Na}_{2} \mathrm{CO}_{3}}}{\mathrm{NH}_{2}}$

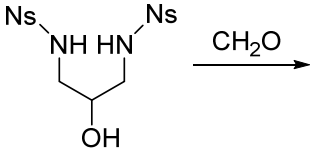

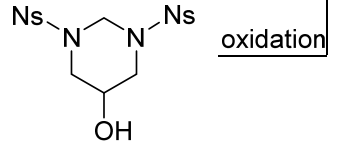

图式 32 化合物 $\mathbf{1 1 7}$ 的合成

Scheme 32 Synthesis of $\mathbf{1 1 7}$
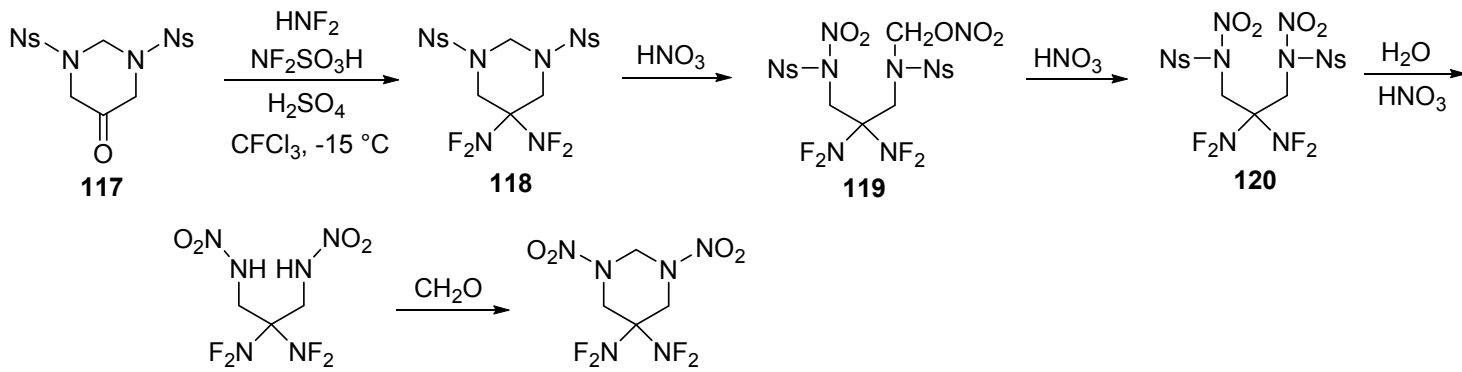

121

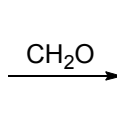

RNFX

图式 33 RNFX 的合成

Scheme 33 Synthesis of RNFX
基甲基-3-甲基环氧丁烷(122)和 3,3-双(氨基甲基)环氧丁 烷(126)为母体, 通过取代、氟化反应率先合成出了单二 氟氨甲基取代的环氧丁烷化合物 124 和双二氟氨甲基取 代的环氧丁烷化合物 $\mathbf{1 2 8}$, 在硝酸的二氯甲烷溶液对其 硝化开环, 获得了同时具有硝酸酯基和二氟氨甲基结构 的高能增塑剂 2-双 (二氟氨甲基)-1,3-丙二醇二硝酸酯 (125)和 2-二氟氨甲基-2-甲基-1,3-丙二醇二硝酸酯(129), 合成路线如 Scheme 34 所示.

2015 年, 南京理工大学潘仁明等 ${ }^{[102]}$ 对化合物 $\mathbf{1 2 4}$ 的合成进行了优化, 以三差圣基乙烷为原料, 经溴代酯 化、碱关环、高压胺化、酰胺保护和低温氟化五步反应, 得到了化合物 124, 总收率为 $43 \%$, 获得了经济可行的 合成工艺. 2016 年, 该课题组 ${ }^{[103]}$ 完成了化合物 124 在浓 硝酸、硝硫混酸、硫酸/硝酸钾及 $\mathrm{N}_{2} \mathrm{O}_{5}$ 体系下开环硝化 合成化合物 125 的工艺，使开环硝化收率提高到 $65 \%$; 差示扫描量热(DSC) 实验表明化合物 125 起始分解温度
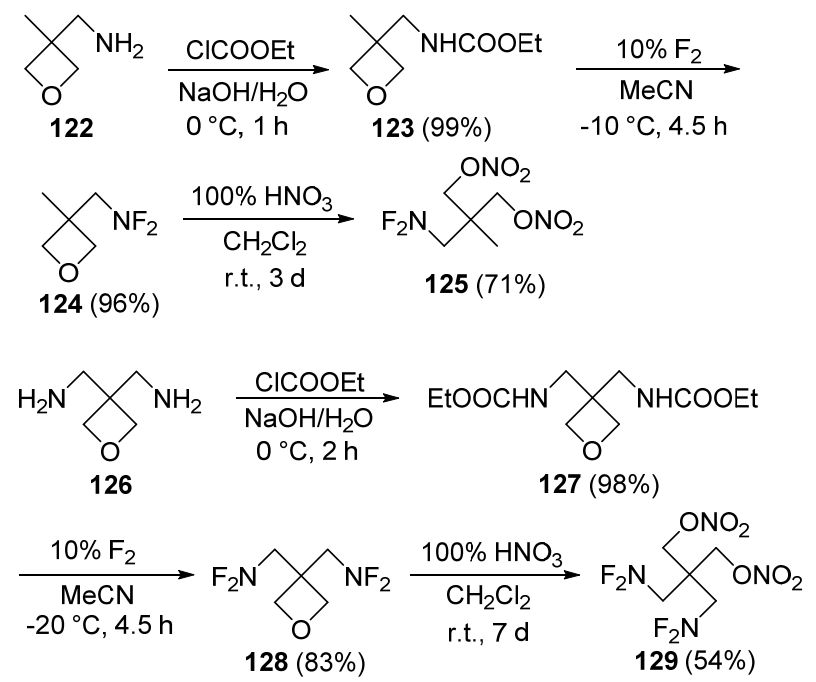

图式 34 化合物 125 和 129 的合成 Scheme 34 Synthesis of $\mathbf{1 2 5}$ and $\mathbf{1 2 9}$ 
为 $162.6{ }^{\circ} \mathrm{C}$, 分解峰温为 $208.0{ }^{\circ} \mathrm{C}$, 同时还测定了该化 合物与部分材料相容性, 结果显示化合物 125 与 CL-20、 $\mathrm{AP}$ 及 $\mathrm{Al}$ 等均相容，与 RDX、HMX 及 $\mathrm{B}$ 不相容.

2012 年, 张明权等 ${ }^{[43]}$ 完成了化合物 129 合成工艺优 化和性能研究, 以 3,3-二氯甲基氧杂环丁烷为原料, 通 过氨化、酯化、氟化反应完成了化合物 129 的合成, 总 收率约 $43 \%$. 热分析表明, 化合物 $\mathbf{1 2 9}$ 熔点为 $43.5{ }^{\circ} \mathrm{C}$, 在 15 200 ${ }^{\circ} \mathrm{C}$ 范围内不发生分解, 热稳定性良好; 撞击 感度 $H_{50} \approx 28.5 \mathrm{~cm}(5 \mathrm{~kg}$ 落锤), 摩擦感度为 $100 \%$ (摆角 $90^{\circ}$, 压力 $9.92 \mathrm{MPa}$ ). 同年, 潘仁明等 ${ }^{[42]}$ 采用 GJB 772A-97 502.1 完成了化合物 129 与典型炸药、推进剂组 分的化学相容性. 结果表明, 化合物 129 与 RDX、 HMX、2,6-二氨基-3,5-二硝基-1-氧-吡嗪(LLM-105)、高 氯酸铵(AP)、碱式碳酸铅的化学相容性达到 1 级, 与 $3,4-$ 二硝基呋咱基氧化呋咱(DNTF)、六硝基茷(HNS)、 $\mathrm{B}$ 粉、 1 号中定剂(二甲基二苯脲)的化学相容性达到 2 级, 与 TNT、硝化棉(NC)-硝化甘油(NG)、Al 化学不相容.

\section{2 二氟氨基聚合物}

由于二氟氨基对推进剂比冲提升效果显著, 作为含 能粘合剂使用的二氟氨基类聚合物早在 20 世纪 60 年代 初便开始了研制工作, 由于结构不稳定以及合成难度大 等问题, 并未得到实质发展. 直到 1995 年, Manser 等 ${ }^{[104]}$ 在合成 124 和 128 单体基础上, 以二氯甲烷为溶剂, 1,4丁二醇/三氟化棚乙醚为催化引发剂, 成功制备出 $\mathbf{1 2 4}$ 、

128 的均聚物以及 $124 \sim 128$ 共聚物, 如 Scheme 35 所示. 均聚物 131 由于结构对称性好, 导致其结晶显著, 玻璃 化转变温度 $\left(T_{\mathrm{g}}\right)$ 为 $138{ }^{\circ} \mathrm{C}$, 熔点为 $158{ }^{\circ} \mathrm{C}$, 初始分解温 度为 $210{ }^{\circ} \mathrm{C}$. 均聚物 130 以及共聚物 132 结构对称性明 显降低, 在常温下均是液态, 均聚物 $\mathbf{1 3 0}$ 玻璃化转变温 度为 $-21{ }^{\circ} \mathrm{C}$, 起始分解温度为 $191{ }^{\circ} \mathrm{C}$, 共聚物 132 起 始分解温度为 $192{ }^{\circ} \mathrm{C}$, 二者作为含能粘合剂使用均有 一定的优势.

2015 年, 潘仁明课题组 ${ }^{[102]}$ 开展了催化剂用量和反 应温度对单体 124 聚合反应的影响, 获得的开环均聚优 化工艺为: 三氟化硼乙醚(TFBE)为阳离子催化剂, 1,4丁二醇 $(\mathrm{BDO})$ 为引发剂, 聚合反应温度为 $-10{ }^{\circ} \mathrm{C}$, 反应 时间为 $24 \mathrm{~h}$, 获得的均聚物 $\mathbf{1 3 0}$ 的平均分子量 $\left(M_{\mathrm{n}}\right)$ 为 3056 , 多分散性指数 $(\mathrm{PDI})$ 为 1.73 , 官能度 $(f)$ 为 $1.87, T_{\mathrm{g}}$ 为 $-40.8{ }^{\circ} \mathrm{C}$. 采用 DSC 法, 研究了共聚物 130 的热分解 行为及动力学, 发现 130 的热分解由两个热失重阶段组 成, 第一个阶段主要是 HF 的两步不完全解离, 第二阶 段则是剩余的单氟亚胺基团与聚合物链段的分解. 利用 DSC 法测得了 130 与火炸药中常见组分的化学相容性, 结果表明, 130 与 TNT、RDX、2,4-二硝基苯甲醚 $(D N A N) 、 P E T N 、 A P 、 A l$ 相容性化为 $\mathrm{A}$ 级; 与 HMX、

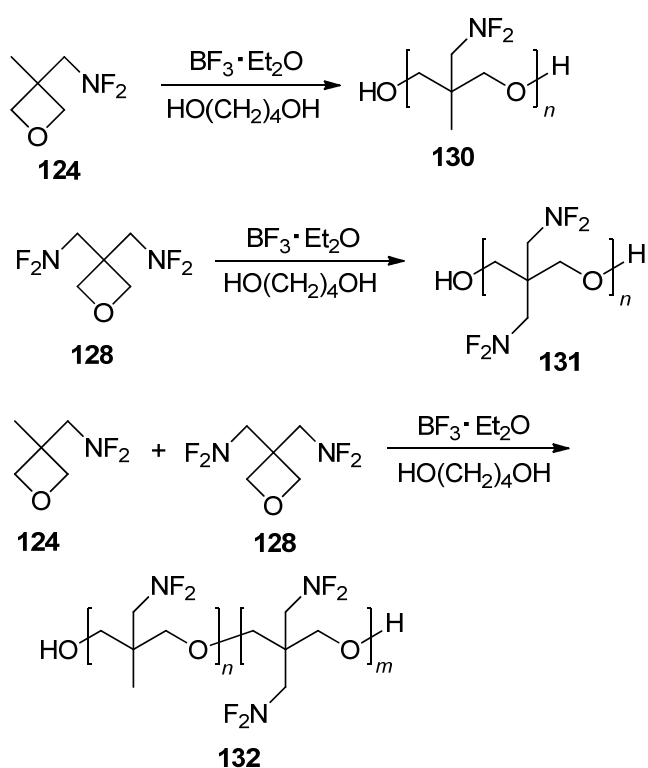

图式 35 二氟氨基聚合物 130, 131 和 132 的合成 Scheme 35 Synthesis of $\mathrm{NF}_{2}$-based polymers 130, 131 and 132 CL-20、3-硝基-1,2,4-三夾-5-酮(NTO)、硝酸铵(AN)、 $\mathrm{Mg} 、 \mathrm{~B}$ 等相容性差, 为 $\mathrm{D}$ 级.

为了进一步提高生成热和氧平衡并有效降低玻璃 化温度, 克服单一均聚物的性能缺陷, 潘仁明课题 组 ${ }^{[102]}$ 分别将 3,3-偕叠氮甲基氧杂环丁烷 (BAMO)、3-偕 叠氮甲基- 3-甲基氧杂环丁烷(AMMO)、3-硝酸酯甲基-3甲基氧杂环丁烷(NIMMO)、THF 分别与 $\mathbf{1 2 4}$ 共聚, 成功 将二氟氨基与叠氮基、硝酸酯结合, 合成出四种新型二 氟氨基共聚物 133、134、135 和 136, 合成路线如 Scheme 36 所示, 并完成了核磁、红外、 $M_{\mathrm{n}} 、 \mathrm{PDI} 、 f$ 及 $T_{\mathrm{g}}$ 等研 究(表 4). 通过 TG/DSC 联用, 得到了共聚物的起始分解 温度 $\left(T_{\text {onset }}\right) 、$ 热分解动力学及机理. 该研究工作原创性 强，获得的新型二氟氨基粘合剂性能突出，具有很高的 学术和应用价值, 将我国二氟氨基粘合剂研究工作推上 新的高度.

表 4 二氟氨基粘合剂 $133,134,135$ 和 136 的参数 Table 4 Parameters of $\mathrm{NF}_{2}$-based binders 133, 134, 135 and 136

\begin{tabular}{cccccc}
\hline Substrate & $T_{\text {onset }}{ }^{\circ} \mathrm{C}$ & $M_{\mathrm{n}} /\left(\mathrm{g} \cdot \mathrm{mol}^{-1}\right)$ & PDI & $f$ & $T_{\mathrm{g}} /{ }^{\circ} \mathrm{C}$ \\
\hline $\mathbf{1 3 3}$ & 190 & 5772 & 1.30 & 1.98 & -24.3 \\
$\mathbf{1 3 4}$ & 175 & 4827 & 1.25 & 1.87 & -38.7 \\
$\mathbf{1 3 5}$ & 125 & 2675 & 1.79 & 2.69 & -28.4 \\
$\mathbf{1 3 6}$ & - & 2700 & 2.11 & 1.88 & -50.8 \\
\hline
\end{tabular}

\section{6 结论与展望}

氟氮含能化合物在增加化合物密度、提升推进剂比 冲以及提高金属粉燃烧效率等方面具有传统硝基含能 化合物无法比拟的优势，已成为开发下一代新型高能量 


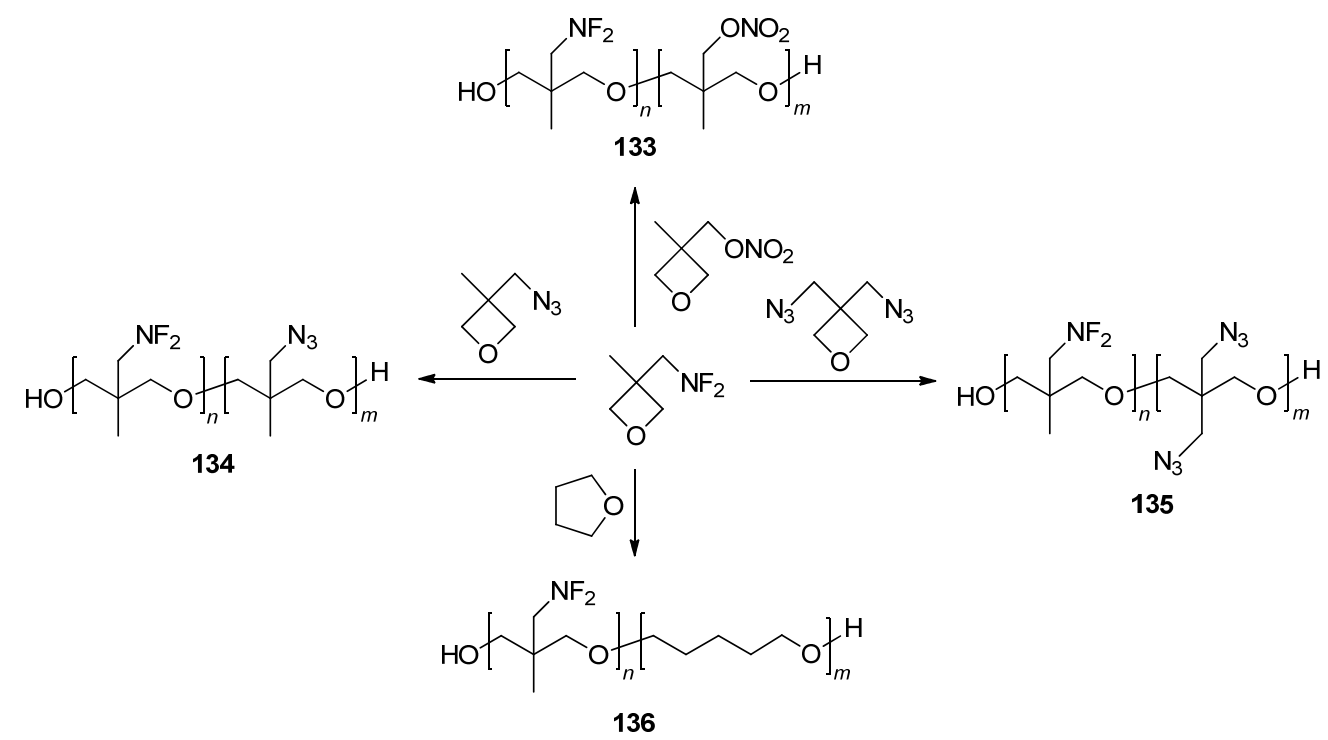

图式 36 二氟氨基粘合剂 $133,134,135$ 和 136 的合成

Scheme 36 Synthesis of $\mathrm{NF}_{2}$-based binders 133, 134, 135 and 136

密度材料的重要途径. 经过研究人员几十年的努力, 氟 氮含能化合物研究取得巨大进步，已经开发出氟氮唑、 二氟氨基二硝甲基、偕二氟氨基硝胺氮杂环等多种类型 高能量密度化合物, 显示出诱人的性能和潜在的应用前 景. 然而，目前仍然面临合成步骤长、反应条件苛刻、 环境污染大以及氟氮化合物感度高、热稳定性不够好等 问题, 导致很难批量生成和实际应用. 因此, 为加快新 型氟氮含能化合物研发速度和提升应用可行性, 建议可 从以下几个方面开展工作: (1)开发结构稳定、安全性好 且高效的新型二氟氨基化试剂, 避免使用稳定性差、易 爆、环境污染大的二氟氨基化试剂, 如 $\mathrm{HNF}_{2} 、 \mathrm{~N}_{2} \mathrm{~F}_{4}$ 等, 打破二氟氨基含能化合物合成条件限制, 加快二氟氨基 含能化合物开发步伐. (2)采用共轭体系、分子内和分子 间氢键、 $\pi-\pi$ 堆积等策略，改善氟氮含能化合物感度高、 热稳定性差的问题，从分子层面实现高能与降感的统 一. (3)进一步探究氟氮含能化合物构效关系, 利用量子 化学手段并结合晶体结构、感度和热性能实验数据, 阐 明 $\mathrm{N}-\mathrm{F}$ 键对氟氮含能化合物密度、感度、热稳定性的 影响规律, 设计合成更高能量密度且结构稳定性好的新 型氟氮化合物提供理论支撑. (4)加强 HNFX、RNFX 等 具有潜在应用前景的氟氮含能化合物合成工艺优化和 新路线探索研究，寻找合成步骤短、安全性好、成本低、 环境污染小的新工艺和新方法, 促进相关化合物性能和 基础应用研究. (5)探索氟氮含能化合物在炸药爆轰和推 进剂燃烧的反应过程和机理, 通过气体产物分析, 推测 氟氮化合物氟原子在爆轰和燃烧过程中反应动力学和 机理, 实现含氟氮化合物的炸药和推进剂配方最优化, 推进应用研究工作.

\section{References}

[1] Gao, H.; Shreeve, J. M. Chem. Rev. 2011, 111, 7377.

[2] Klapötke, T. M. Chemistry of High-energy Materials, 2nd ed., Walter de Gruyter, Berlin, 2012.

[3] Badgujar, D. M.; Talawar, M. B.; Asthana, S. N.; Mahulikar, P. P. J. Hazard. Mater. 2008, 151, 289.

[4] Xue, Q.; Bi, F. Q.; Zhang, J. R.; Zhang, J. L.; Wang, B. Z.; Zhang, S. R. Chin. J. Org. Chem. 2019, 39, 1244 (in Chinese). (薛琪, 毕福强, 张家荣, 张俊林, 王伯周, 张生勇, 有机化学, 2019, 39, 1244.)

[5] Wang, Y.; Liu, Y. J.; Song, S. W.; Yang, Z. J.; Qi, X. J.; Wang, K. C.; Liu, Y.; Zhang, Q. H.; Tian, Y. Nat. Commun. 2018, 9, 2444.

[6] Tang, Y. X.; Kumar, D.; Shreeve, J. M. J. Am. Chem. Soc. 2017, $139,13684$.

[7] Zhai, L. J.; Bi, F. Q.; Huo, H.; Luo, Y. F.; Li, X. Z.; Chen, S. P.; Wang, B. Z. Front. Chem. 2019, 7, 559.

[8] Zhang, J. R.; Bi, F. Q.; Lian, P.; Zhang, J. L.; Wang, B. Z. Chin. J. Org. Chem. 2017, 37, 2736 (in Chinese). (张家荣, 毕福强, 廉鹏, 张俊林, 王伯周, 有机化学, 2017, 37, 2736.)

[9] Li, Y. L.; Xue, M.; Wang J. L.; Cao, D. L.; Ma, Z. L. Chin. J. Org. Chem. 2016, 36, 1528 (in Chinese) (李云路，薛梅，王建龙，曹端林，马忠亮，有机化学， 2016，36, 1528.)

[10] Zhou, J.; Zhang, J. L.; Ding, L.; Bi, F. Q.; Wang, B. Z. Chin. J. Energ. Mater. 2019, 27, 708 (in Chinese). (周静, 张俊林, 丁黎, 毕福强, 王伯周, 含能材料, 2019, 27, 708.)

[11] Zhou, J.; Zhang, J. L.; Ding, L.; Bi, F. Q.; Wang, B. Z. Chin. J. Explos. Propellants 2019, 42, 608 (in Chinese). (冯晓军, 薛乐星, 曹芳洁, 刘谦, 李欣, 火炸药学报, 2019, 42, 608.)

[12] Zhai, L. J.; Bi, F. Q.; Luo, Y. F.; Wang, N. X.; Zhang, J. L.; Wang, B. Z. Sci. Rep. 2019, 9, 4321.

[13] Xu, Y. G.; Shen, C.; Lin, Q. H.; Wang, P. C.; Jiang, C.; Lu, M. J. Mater. Chem. A 2016, 4, 17791.

[14] Barton, L. M.; Edwards, J. T.; Johnson, E. C.; Bukowski, E. J.; Sausa, R. C.; Byrd, E. F. C.; Orlicki, J. A.; Sabatini, J. J.; Baran, P. S. J. Am. Chem. Soc. 2019, 141, 12531.

[15] Zhai, L. J.; Bi, F. Q.; Luo, Y. F.; Sun, L.; Huo, H.; Zhang, J. C.; Zhang, J. L.; Wang, B. Z.; Chen, S. P. Chem. Eng. J. 2020, 391, 123573 
[16] Vishnevskiy, Y. V.; Tikhonov, D.; Schwabedissen, J.; Stammler, H-G.; Moll, R.; Krumm, B.; Klapötke, T. M.; Mitzel, N. W. Angew. Chem., Int. Ed. 2017, 56, 9619.

[17] Yu, Q.; Yin, P.; Zhang, J. H.; He, C. L.; Imler, G. H.; Parrish, D. A.; Shreeve, J. M. J. Am. Chem. Soc. 2017, 139, 8816.

[18] Lukin, K. A.; Li, J.; Eaton, P. E.; Kanomata, N.; Hain. J.; Punzalan, E.; Gilardi, R. J. Am. Chem. Soc. 1997, 119, 9591.

[19] Zhang, M. X.; Eaton, P. E.; Gilardi, R. Angew. Chem., Int. Ed. 2000, 39, 401

[20] Gong, X. B.; Sun, C. H.; Pang, S. P.; Zhang, J.; Li, Y. C.; Zhao, X. Q. Chin.J. Org. Chem. 2012, 32, 486 (in Chinese). (公绪滨, 孙成辉, 庞思平, 张静, 李玉川, 赵信岐, 有机化学, 2012, 32, 486.)

[21] Nair, U. R.; Sivabalan, R.; Gore, G. M.; Geetha, M.; Asthana S. N.; Singh, H. Combust., Explos. Shock Waves 2005, 41, 121.

[22] Ammon, H. L. Struct. Chem. 2001, 12, 205.

[23] Feng, Z. G. Prog. Chem. 2000, 12, 171 (in Chinese). (冯增国, 化学进展, 2000, 12, 171.)

[24] Liu, H.; Zhang, Y. J.; Zhang, L. Y.; Zheng, W. F.; Pan, R. M. Chin. J. Explos. Propellants 2019, 42, 363 (in Chinese). (刘卉, 张英杰, 张路遥, 郑文芳, 潘仁明, 火炸药学报, 2019, 42, 363.)

[25] Yetter, R. A.; Dryer, F. L.; Rabitz, H.; Brown, R. C.; Kolb, C. E. Combust. Flame 1998, 112, 387.

[26] http: //webbook.nist.gov/chemistry/

[27] Valluri, S. K.; Schoenitz, M.; Dreizin, E. Def. Technol. 2019, 15, 1.

[28] Li, S. W.; Zhao, F. Q.; Yuan, C.; Luo, Y.; Gao, Y. J. Solid Rocket Technol. 2002, 25, 36 (in Chinese). (李上文, 赵凤起, 袁潮, 罗阳, 高茵, 固体火箭技术, 2002, 25, 36.)

[29] Chapman, R. D. Struct. Bonding 2007, 125, 123.

[30] Li, H.; Qin, Y. J.; Li, J. H.; Pan, R. M.; Wang, W. J. Chem. Bull. 2012, 75, 1076 (in Chinese). (李欢, 秦叶军, 李金华, 潘仁明, 王万军, 化学通报, 2012, 75, 1076.)

[31] Klapötke, T. M. J. Fluorine Chem. 2006, 127, 679.

[32] Chen, J. F.; Yu, Y.; Li, Y. C.; Pang, S. P. J. Fluorine Chem. 2018, $205,35$.

[33] Ruff, O.; Giese, M. Eur. J. Inorg. Chem. 1936, 69, 598.

[34] Davenas, A. J. Propul. Power 2003, 19, 1108.

[35] Petry, R. C.; Freeman, J. P. J. Org. Chem. 1967, 32, 4034

[36] Coon, C. L.; Ross, D. L. US 3732288, 1973.

[37] Reed, S. F.; Shoults, R. D. J. Org. Chem. 1972, 37, 3326.

[38] Coon, C. L.; Ross, D. L. US 3714254, 1973.

[39] Wiener, C.; Tyler, W. E. US 4128583, 1978.

[40] Zheng, Y. Y.; Zhou, J. Z.; Zhou, D. L.; Zhang, M. N. Acta Armamentarii 1988, 1, 59 (in Chinese). (郑远洋, 周继璋, 周大㐘, 张明南, 兵工学报, 1988, 1, 59.)

[41] Wang, W. J.; Li, H.; Pan, R. M.; Zhu, W. H. Chin. J. Org. Chem. 2019, 39, 170 (in Chinese). (王万军, 李 欢, 潘仁明, 朱卫华, 有机化学, 2019, 39, 170.)

[42] Li, H.; Zhang, L. Y.; Pan, R. M.; Wang, W. J. Chin. J. Explos. Propellants. 2012, 35, 37 (in Chinese). (李欢, 张路遥, 潘仁明, 王万军, 火炸药学报, 2012, 35, 37.)

[43] Zhang, M. Q.; Liu, H. Y.; Gao, B. Z.; Zhang, L.; Kang, L.; Zhang, K. R. Chin. J. Energ. Mater. 2012, 20, 314 (in Chinese). (张明权, 刘红雨, 高宝柱, 张否, 康玲, 张可人, 含能材料, 2012, 20, 314.)

[44] Laali, K. K.; Tanaka, M.; Forohar, F.; Cheng, M.; Fetzer, J. C. J. Fluorine Chem. 1998, 91, 185.

[45] Dalinger, I. L.; Shkineva, T. K.; Vatsadze, I. A.; Popova, G. P.; Shevelev, S. A. Mendeleev Commun. 2011, 21, 48.

[46] Grakauskas, V.; Baum, K. J. Org. Chem. 1969, 34, 2840.

[47] Chapman, R. D.; Davis, M. C.; Gilardi, R. Synth. Commun. 2003, 35,4173 .

[48] Sharts, C. M. J. Org. Chem. 1968, 33, 1008.

[49] Mcpake, C. B.; Murray, C. B.; Sandford, G. Aust. J. Chem. 2013, 66,145 .
[50] Archibald, T. G.; Manser, G. E. US 5789617, 1998.

[51] Emele'us, H. J.; Shreeve, J. M.; Verma, R. D. Adv. Inorg. Chem. 1989, 33, 139.

[52] Klapdor, M. F.; Willner, H.; Poll, W.; Mootz, D. Angew. Chem. 1996, $108,320$.

[53] Freeman, J. P.; Kennedy, A.; Colburn, C. B. J. Am. Chem. Soc. 1960, 82,5304

[54] Petry, R. C.; Freeman, J. P. J. Am. Chem. Soc. 1961, 83, 3912.

[55] Graham, W. H.; Parker, C. O. J. Org. Chem. 1963, $28,850$.

[56] Grakauskas, V.; Baum, K. J. Am. Chem. Soc. 1970, 92, 2096.

[57] Banks, R. E.; Haszeldine, R. N.; Lalu, J. P. J. Chem. Soc., C 1966, 1514.

[58] Baum, K. J. Am. Chem. Soc. 1968, 90, 7083.

[59] Fokin, A. V.; Kosyrev, Y. M.; Shevchenko, V. I. Russ. Chem. Bull. 1983, 31, 1626.

[60] Graham, W. H.; Freeman, J. P. J. Am. Chem. Soc. 1967, 89, 716.

[61] Keith, J. N.; Douthart, R. J.; Sumida, W. K.; Solomon, I. J. Advanced Propellant Chemistry 1966, 141.

[62] Haiges, R.; Wagner, R.; Boatz, J. A.; Yousufuddin, M.; Etzkorn, M.; Surya Prakash, G. K.; Christe, K. O.; Chapman, R. D.; Welker, M. F.; Kreutzberger. C. B. Angew. Chem., Int. Ed. 2006, 45, 5179.

[63] Coon, C. L.; Hill, M. E.; Ross, D. L. US 3759998, 1973

[64] Baum, K.; Grakauskas, V. US 4075246, 1978.

[65] Flanagan, G. E.; Frankel, M. B.; Witucki, E. F. US 4141910, 1979.

[66] Surya Prakash, G. K.; Etzkorn, M.; Olah, G. A.; Christe, K. O.; Schneidera, S.; Vij, A. Chem. Commun. 2002, 1712.

[67] Lustig, M.; Cady, G. H. Inorg. Chem. 1963, 2, 388.

[68] Fokin, A. K.; Studnev, Y. N.; Rapkin, A. L.; Kuznetsova, L. D. Russ. Chem. Bull. 1996, 45, 2547.

[69] Dalinger, I. L.; Shakhnes, A. K.; Monogarov, K. A.; Suponitsky, K. Y.; Sheremetev, A. B. Mendeleev Commun. 2015, 25, 429.

[70] Semenov, V. V.; Shevelev, S. A.; Bruskin, A. B.; Shakhnes, A. K.; Kuz'min, V. S. Chem. Heterocycl. Compd. 2017, 53, 728.

[71] Dalinger, I. L.; Kormanov, A. V.; Suponitsky, K. Yu.; Muravyev, N. V.; Sheremetev, A. B. Chem. Asian J. 2018, 13, 1165.

[72] Colburn, C. B.; Kennedy, A. J. Am. Chem. Soc. 1958, 80, 5004.

[73] Baumgardner, C. L.; Lawton, E. L. Acc. Chem. Res. 1974, 7, 14

[74] Reed Jr, S. F. J. Org. Chem. 1968, 33, 1861

[75] Petry, R. C.; Parker, C. O.; Johnson, F. A.; Stevens, T. E.; Freeman, J. P. J. Org. Chem. 1967, 32, 1534

[76] Petry, R. C.; Freeman, J. P. J. Am. Chem. Soc. 1961, 83, 3912.

[77] Gakh, A. A.; Romaniko, S. V.; Ugrak, B. I.; Fainzilberg, A. A. Tetrahedron 1991, 47, 7447.

[78] Majumder, U.; Armantrout, J. R.; Williams, R. V.; Shreeve, J. M. J. Org. Chem. 2002, 67, 8435.

[79] Belter, R. K. J. Fluorine Chem. 2012, 132, 961.

[80] Dalinger, I. L.; Vinogradov, V. M.; Shevelev, S. A.; Kuz'min, V. S.; Arnautova, E. A.; Pivina, T. S. Propellants, Explos., Pyrotech. 1998, 23, 212

[81] Fokin, A. K.; Studnev, Y. N.; Stolyarov, V. P.; Mel'nikov, A. A. Russ. Chem. Bull. 2000, 49, 949.

[82] Marsden, H. M.; Shreeve, J. M. Inorg. Chem. 1987, 26, 169

[83] John, E. O.; Shreeve, J. M. Inorg. Chem. 1988, 27, 3100.

[84] John, E. O.; Kirchmeier, R. L.; Shreeve, J. M. J. Fluorine Chem. 1990, 47, 333

[85] John, E. O.; Willett, R. D.; Scott, B.; Kirchmeier, R. L.; Shreeve, J. M. Inorg. Chem. 1989, 28, 893.

[86] Ye, C. f.; Gao, H. X.; Shreeve, J. M. J. Fluorine Chem. 2007, 128, 1410 .

[87] Litvinov, B. V.; Fainzil'berg, A. A.; Pipekin, V. I.; Smirnov, S. P.; Loboiko, B. G.; Shevelev, S. A.; Nazin, G. M. Dokl. Akad. Nauk 1994, 336, 67

[88] Khisamutdinov, G. K.; Shevelev, S. A. Russ. Chem. Bull., Int. Ed. 2001, 50, 736 .

[89] Fokin, A. V.; Studnev, Y. N.; Kuznetsova, L. D. Dokl. Akad. Nauk 1996, 3,358

[90] Fokin, A. V.; Studnev, Y. N.; Kuznetsova, L. Russ. Chem. Bull. 1996, 45, 1952 . 
[91] Zhang, M. Q.; Liu, H. Y;; Wei, X. C.; Zhang, L.; Kang, L. Chem. Propellants Polym. Mater. 2017, 15, 45 (in Chinese).

(张明权, 刘红雨, 韦兴存, 张否, 康玲, 化学推进剂与高分子材 料, 2017, 15, 45.)

[92] Frankel, M. B.; Witucki, E. F. US 4341712, 1982.

[93] Adolph, H. G.; Trivedi, N. J. US 6325876 B1, 2001.

[94] Ammon, H. L.; Holden, J. R.; Du, Z. Structure and Density Predictions for Energetic Materials, 2002. http://www.chem.missouri.edu/ thompson/MURI02/extended/Ammon_MURI_extended_abstract_ 4.pdf.

[95] Baum, K.; Trivedi, N. J.; Lovato, J. M.; Iyer, V. K. Report NRO-1-1 (final), Fluorochem, Azusa, CA, 1993.

[96] Chapman, R. D.; Welker, M. F; Kreutzberger, C. B. J. Org. Chem. 1998, 63, 1566.
[97] Chapman, R. D.; Gilardi, R. D.; Welker, M. F.; Kreutzberger, C. B. J. Org. Chem. 1999, 64, 960.

[98] Chapman, R. D.; Groshens, T. J. US 7563889 B1, 2009.

[99] Chapman, R. D.; Groshens, T. J. US 8444783 B1, 2013.

[100] Axenrod, T.; Guan, X. P.; Sun, J. G.; Qi, L.; Chapman, R. D.; Gilardi, R. D. Tetrahedron Lett. 2001, 42, 2621.

[101] Chapman, R. D.; Nguyen, B. V. US 6310204 B1, 2001.

[102] Li, H. Ph.D. Dissertation, Nanjing University of Science \& Technology, Nanjing, 2015 (in Chinese). (李欢, 博士论文, 南京理工大学, 南京, 2015.)

[103] Mei, Y. M.S. Thesis, Nanjing University of Science \& Technology, Nanjing, 2016 (in Chinese). (梅莹, 硕士论文, 南京理工大学, 南京, 2016.)

[104] Archibald, T. G.; Manser, G. E. Immoos, J. E. US 5240311, 1995.

(Li, L.; Fan, Y.) 